\title{
General conformable estimators with finite-time stability
}

\author{
Fidel Meléndez-Vázquez ${ }^{1 *}$ [D, Guillermo Fernández-Anaya and Eduardo G. Hernández-Martínez²
}

\author{
"Correspondence: \\ asistente.postdoc02@ibero.mx \\ 'Physics and Mathematics \\ Department, Universidad \\ Iberoamericana, Prol. Paseo de la \\ Reforma 880, Lomas de Santa Fe, \\ Álvaro Obregón, 01219 México City, \\ México \\ Full list of author information is \\ available at the end of the article
}

\begin{abstract}
In this paper, some estimators are proposed for nonlinear dynamical systems with the general conformable derivative. In order to analyze the stability of these estimators, some Lyapunov-like theorems are presented, taking into account finite-time stability. Thus, to prove these theorems, a stability function is defined based on the general conformable operator, which implies exponential stability. The performance of the estimators is assessed by means of numerical simulations. Furthermore, a comparison is made between the results obtained with the integer, fractional, and general conformable derivatives.
\end{abstract}

Keywords: General conformable derivative; General conformable exponential stability; Conformable estimator; Finite-time stability

\section{Introduction}

Fractional calculus, the generalization of calculus to noninteger orders, besides looking to extend the classical mathematical results, has had many applications to physical systems since the 1970s of the twentieth century. The first results in this area involved the Riemann-Liouville integral and the Riemann-Liouville and Caputo derivatives, which are still widely studied and used [1-8]. Nevertheless, other definitions of noninteger operators have been developed; some recent classifications of the main operators and their properties appear in $[9,10]$.

Given that the Riemann-Liouville and Caputo derivatives may deal with singularity issues in the kernel, some operators with nonsingular kernel have been proposed, such as Caputo-Fabrizio [11] and Atangana-Baleanu [12] derivatives; these operators are currently being studied extensively, and they have been used for both theoretical results and applications [13-17].

Furthermore, another reason for proposing other noninteger operators is that the Riemann-Liouville and Caputo derivatives do not satisfy the main results of classical calculus, such as the Leibniz product rule, the chain rule, the semigroup property, and the fundamental theorem, which would be expected to occur naturally for their use in applications. In this sense, Khalil et al. proposed the so-called conformable derivative [18]; this operator satisfies the properties mentioned and other mathematical results. Later, Abdeljawad used this definition to extend more results in calculus and linear systems [19].

(c) The Author(s) 2020. This article is licensed under a Creative Commons Attribution 4.0 International License, which permits use sharing, adaptation, distribution and reproduction in any medium or format, as long as you give appropriate credit to the original author(s) and the source, provide a link to the Creative Commons licence, and indicate if changes were made. The images or other third party material in this article are included in the article's Creative Commons licence, unless indicated otherwise in a credit line to the material. If material is not included in the article's Creative Commons licence and your intended use is not permitted by statutory regulation or exceeds the permitted use, you will need to obtain permission directly from the copyright holder. To view a copy of this licence, visit http://creativecommons.org/licenses/by/4.0/. 
After that, many papers have been focused on studying the theory and applications of this derivative [20-25].

Similarly to Khalil, Katugampola proposed some conformable-type operators, which also satisfy the classical results [26]. These operators have also been studied and applied [27-30].

Moreover, Akkurt et al. proposed the so-called generalized fractional derivative [31], which generalizes the operators defined by Khalil and Katugampola, while still satisfying the results from integer calculus. This derivative is called general due to the freedom of choice of its kernel $k(t)$, where its adequate selection permits to obtain other operators as particular cases.

Additionally, Zhao and Luo defined another general version, called the general conformable derivative (GCD) [32], which is based on the linear extended Gâteaux derivative. This operator also generalizes the Khalil and Katugampola derivatives, but in this case the kernel not only depends on time, but also on the order $\alpha$. This operator also satisfies the desired results from classical calculus and encompasses other derivatives as special cases.

On the other hand, the problem of finite-time stability becomes relevant in applications of dynamical systems, where theoretical asymptotic stability is not useful, but it is desired that the system trajectories reach the equilibrium in a determined finite time. Thus, this theme has been studied and developed formally [33-36]; some applications and specific problems can be found in [37-39]. Moreover, this subject has been addressed for fractional-order systems [40-42] and even for systems with conformable derivatives [4345]. However, it has been reported that, in general, nonlinear fractional-order systems cannot have finite-time stability, but just under certain considerations [46, 47].

Given that the GCD generalizes other operators and satisfies the classical calculus rules, it is of great interest to use it to develop results in theory and applications, which would enclose existing or possible results using conformable-type derivatives. Hence, in this paper, some nonlinear estimators for dynamical systems that involve the GCD are proposed. In order to verify the stability of these estimators, an exponential-like function is defined, based on the operator in question, which implies exponential stability. Using this function, some Lyapunov-like theorems are proven; then, some finite-time stability conditions are added to these theorems. The performance of the designed estimators is evaluated with numerical simulations; for this, the models considered are a mechanical system and a chaotic oscillator.

This work is divided as follows. In Sect. 2, the GCD is defined from the original conformable derivative; its properties and some results are presented. In Sect. 3, the so-called general conformable exponential function is defined, and then some Lyapunov-like theorems for systems with the GCD are proven. Section 4 presents some definitions and conditions required for finite-time stability, and the Lyapunov-like theorems previously defined are extended to consider them. In Sect. 5, a pair of nonlinear estimators with general conformable dynamics are designed, and they are proven to be finite-time stable in the general conformable sense. In Sect. 6, numerical simulations are used to assess the performance of the estimators; the procedure is applied to the general conformable models of the simple pendulum and the Van der Pol oscillator, and a comparison is made with their integer and fractional versions. Finally, conclusions and results are discussed in Sect. 7.

\section{General conformable derivative}

In this section, some definitions are presented in order to introduce the GCD. 
Definition 1 ([18]) Given a function $f:[0, \infty) \rightarrow \mathbb{R}$, the conformable derivative (CD) of $f$ of order $\alpha$ is defined by

$$
T_{\alpha}(f)(t)=\lim _{\varepsilon \rightarrow 0} \frac{f\left(t+\varepsilon t^{1-\alpha}\right)-f(t)}{\varepsilon}
$$

$\forall t>0, \alpha \in(0,1)$. If $\mathrm{f}$ is $\alpha$-differentiable in some $(0, a), a>0$, and $\lim _{t \rightarrow 0^{+}} f^{(\alpha)}(t)$ exists, then define

$$
f^{(\alpha)}(0)=\lim _{t \rightarrow 0^{+}} f^{(\alpha)}(t)
$$

In order to extend and give a physical and geometrical interpretation to this derivative, the following definitions are required.

Definition 2 ([48]) Let $F: U \rightarrow \mathbb{R}$ be a functional, where $U$ is a Banach space. We define the space of admissible variations for $F$, denoted by $\mathcal{V}$, as follows:

$$
\mathcal{V}=\{\psi \mid u+\psi \in U, \forall u \in U\}
$$

Definition 3 ([48]) Given $F: U \rightarrow \mathbb{R}$, we define the Gâteaux variation of $F$ at $u \in U$ on the direction $\psi \in \mathcal{V}$, denoted by $\delta F(u, \psi)$, as follows:

$$
\delta F(u, \psi)=\lim _{\varepsilon \rightarrow 0} \frac{F(u+\varepsilon \psi)-F(u)}{\varepsilon}
$$

if such a limit is well defined. Furthermore, if there exists $u^{*} \in U^{*}$ such that

$$
\delta F(u, \psi)=\left\langle\psi, u^{*}\right\rangle_{U}, \quad \forall \psi \in U,
$$

we say that $F$ is Gâteaux differentiable at $u \in U$, and $u^{*} \in U^{*}$ is said to be the Gâteaux derivative of $F$ at $u$, where $\langle\cdot, \cdot\rangle_{U}$ is the duality pairing between $U$ and $U^{*}$. Finally, we denote

$$
u^{*}=\delta F(u)=\frac{\partial F(u)}{\partial u} .
$$

Remark 1 ([49]) If $F=f \in \mathcal{C}^{1}\left(\mathbb{R}^{n}\right)$ and $u, \psi \in \mathbb{R}^{n}$, then

$$
\delta f(u, \psi)=\lim _{\varepsilon \rightarrow 0} \frac{f(u+\varepsilon \psi)-f(u)}{\varepsilon}
$$

is just the directional derivative of $f$ when $\psi$ is a unit vector. Thus we have that

$$
\delta f(u, \psi)=\nabla f(u) \cdot \psi
$$

and this holds $\forall \psi \in \mathbb{R}^{n}$.

Some properties of the Gâteaux derivative are as follows [32]:

1. $d(c)=0$ (differential of a constant). 
2. $d(f+g)(u, \psi)=d f(u, \psi)+d g(u, \psi)$ (sum rule).

3. $d(f g)(u, \psi)=d f(u, \psi) g+d g(u, \psi) f$ (product rule).

4. $d(f / g)(u, \psi)=\frac{d f(u, \psi) g-d g(u, \psi) f}{g^{2}}$ (quotient rule).

5. $d(f \circ g)(u, \psi)=d f(g(u), d g(u, \psi))$ (chain rule).

Definition 4 ([32]) Suppose that $X$ and $Y$ are locally convex topological vector spaces, $U \subset X$ is open, $f: X \rightarrow Y$, and $\psi(u, \varepsilon, \alpha): X \times \mathbb{R} \times \mathbb{R} \rightarrow X$, where $\alpha \in \mathbb{R}$ is a parameter. The extended Gâteaux differential (EGD) $d f(u, \psi)$ of $f$ at $u \in U$ is defined as

$$
d f^{E G}(u, \psi)=\lim _{\varepsilon \rightarrow 0} \frac{f(u+\psi(u, \varepsilon, \alpha))-f(u)}{\varepsilon}
$$

if the limit exists.

Definition 5 ([32]) Suppose that $X$ and $Y$ are locally convex topological vector spaces, $U \subset X$ is open, $f: X \rightarrow Y$, and $\psi(u, \alpha): X \times \mathbb{R} \rightarrow X$, where $\alpha \in \mathbb{R}$ is a parameter. The linear extended Gâteaux differential (LEGD) $d f(u, \psi)$ of $f$ at $u \in U$ is defined as

$$
d f^{L E G}(u, \psi)=\lim _{\varepsilon \rightarrow 0} \frac{f(u+\varepsilon \psi(u, \alpha))-f(u)}{\varepsilon}
$$

if the limit exists.

The LEGD satisfies the same properties of the Gateaux derivative.

Moreover, regarding the concept of local fractional derivative (LFD), the following principles need to be considered [32]:

P1. LFD should degenerate to the usual first-order derivative when the fractional order equals one.

P2. LFD should have properties consistent with the classical derivative as much as possible.

P3. LFD should have clear physical or geometrical interpretations.

The LEGD satisfies P2 and P3. In order to satisfy P1, the following definition is introduced.

Definition 6 ([32]) A continuous real function $\psi(t, \alpha)$ is called a conformable function if it satisfies

$$
\begin{aligned}
& \psi(t, 1)=1, \\
& \psi(\cdot, \alpha) \neq \psi(\cdot, \beta), \quad \text { where } \alpha \neq \beta \text { and } \alpha, \beta \in(0,1] .
\end{aligned}
$$

Finally, the definition of the operator used in this work is presented.

Definition 7 ([32]) Let $\psi(t, \alpha)$ be a conformable function and $\alpha \in(0,1]$. The general conformable derivative (GCD) is defined as

$$
{ }^{\psi} D^{\alpha} f(t)=\lim _{\varepsilon \rightarrow 0} \frac{f(t+\varepsilon \psi(t, \alpha))-f(t)}{\varepsilon}
$$

if the limit exists. 
Remark 2 Note that

a) when $\psi(t, \alpha)=1,{ }^{\psi} D^{\alpha} f(t)$ degenerates to the usual first-order derivative.

b) when $\psi(t, \alpha)=t^{1-\alpha},{ }^{\psi} D^{\alpha} f(u)$ coincides with the Khalil CD definition.

Moreover, consider the operator proposed by Katugampola.

Definition 8 ([26]) Let $f:[0, \infty) \rightarrow \mathbb{R}$ and $t>0$. Then the derivative of $f$ of order $\alpha$ is defined by

$$
\mathcal{D}^{\alpha}(f)(t)=\lim _{\varepsilon \rightarrow 0} \frac{f\left(t e^{\varepsilon t^{-\alpha}}\right)-f(t)}{\varepsilon}
$$

for $t>0, \alpha \in(0,1)$. If $f$ is $\alpha$-differentiable in some $(0, a), a>0$, and $\lim _{t \rightarrow 0^{+}} \mathcal{D}^{\alpha}(f)(t)$ exists, then define

$$
\mathcal{D}^{\alpha}(f)(0)=\lim _{t \rightarrow 0^{+}} \mathcal{D}^{\alpha}(f)(t) .
$$

Remark 3 When $\psi(t, \varepsilon, \alpha)=t e^{\varepsilon t^{-\alpha}}-t$ in the EGD, it degenerates to the Katugampola definition. However, since $t e^{\varepsilon t^{-\alpha}}-t=\varepsilon t^{1-\alpha}+o\left(\varepsilon^{2}\right)$, it coincides with the Khalil definition neglecting the term $o\left(\varepsilon^{2}\right)$.

Remark 4 The GCD satisfies the same properties as the LEGD, as well as Rolle's and mean value theorems [32].

Remark 5 Henceforth, a function is said to be $\alpha$-differentiable if it is differentiable in the sense of the GCD, with order $\alpha$.

Remark 6 If $f$ is $\alpha$-differentiable, then ${ }^{\psi} D^{\alpha} f(t)=\psi(t, \alpha) \frac{d}{d t} f(t)$.

Theorem 1 ([32]) If a function $f: \mathbb{R}^{+} \rightarrow \mathbb{R}$ is $\alpha$-differentiable at $t>0, \alpha \in(0,1]$, then $f$ is continuous at $t$.

Furthermore, define the inverse operator of the GCD.

Definition 9 ([32]) Let $t \geq a \geq 0, f$ be a function defined on $(a, t]$. Then the $\alpha$-order general conformable integral of $f$ is defined as

$$
{ }^{\psi} I_{a}^{\alpha} f(t)=\int_{a}^{t} \frac{f(\tau)}{\psi(\tau, \alpha)} d \tau
$$

if the Riemann integral exists.

Moreover, consider the following result.

Lemma 1 Let $f:[a, \infty) \rightarrow \mathbb{R}$ be $\alpha$-differentiable on $(a, \infty)$. If ${ }^{\psi} D^{\alpha} f(t) \geq 0$ (respectively $\leq 0) \forall t \in(a, \infty)$, then $f$ is an increasing (respectively decreasing) function.

Proof The proof of this lemma follows from the mean value theorem for the GCD [32]. 
Remark 7 Let $f:[a, \infty) \rightarrow \mathbb{R}$ be $\alpha$-differentiable on $(a, \infty)$. Then

$$
{ }^{\psi} D^{\alpha}\left[f^{2}(t)\right]=2 f(t)^{\psi} D^{\alpha} f(t), \quad \forall t>a
$$

Remark 8 Let $f:[a, \infty) \rightarrow \mathbb{R}^{n}$ be $\alpha$-differentiable on $(a, \infty)$. Then

$$
{ }^{\psi} D^{\alpha}\left[f^{T}(t) f(t)\right]=2 f^{T}(t)^{\psi} D^{\alpha} f(t), \quad \forall t>a .
$$

Remark 9 Let $x:[a, \infty) \rightarrow \mathbb{R}^{n}$ be $\alpha$-differentiable on $(a, \infty)$. Let $P$ be a symmetric positive definite matrix. Then

$$
{ }^{\psi} D^{\alpha}\left[x^{T}(t) P x(t)\right]=2 x^{T}(t) P^{\psi} D^{\alpha} x(t), \quad \forall t>a .
$$

\section{General conformable exponential stability}

Consider the following class of nonlinear systems:

$$
{ }^{\psi} D^{\alpha} x=f(t, x), \quad t>t_{0}, x\left(t_{0}\right)=x_{0},
$$

where $x \in \mathbb{R}^{n}, f: \mathbb{R}^{+} \times \mathbb{R}^{n} \rightarrow \mathbb{R}^{n}$ is a given nonlinear function satisfying $f(t, 0)=0 \forall t \geq 0$ and $\alpha \in(0,1)$.

Definition 10 The origin of system (1) is said to be

i) stable if, for every $\varepsilon>0$ and $t_{0} \in \mathbb{R}^{+}, \exists \delta\left(\varepsilon, t_{0}\right)$ such that, for any $x_{0} \in \mathbb{R}^{n}$, $\left\|x_{0}\right\|<\delta \Longrightarrow\|x(t)\|<\varepsilon, \forall t \geq t_{0}$.

ii) attractive if, for any $t_{0} \geq 0, \exists c\left(t_{0}\right)>0$ such that, for any $x_{0} \in \mathbb{R}^{n}$, $\left\|x_{0}\right\|<c \Longrightarrow \lim _{t \rightarrow \infty} x(t)=0$.

iii) asymptotically stable if it is stable and attractive.

iv) globally asymptotically stable if it is asymptotically stable for any $x_{0} \in \mathbb{R}^{n}$.

The following definition is the essential tool to prove the stability results for this class of systems.

Definition 11 The general conformable exponential function is defined as follows:

$$
E_{\alpha}^{\psi}\left(\gamma, t, t_{0}\right)=\exp \left(\gamma \int_{t_{0}}^{t} \frac{d \tau}{\psi(\tau, \alpha)}\right)
$$

where $\alpha \in(0,1), \gamma \in \mathbb{R}^{+}$and $\psi(t, \alpha) \neq 0$ is a conformable function, $\forall t \geq t_{0}, \forall \alpha \in(0,1)$.

Remark 10 It is not difficult to verify that

$$
{ }^{\psi} D^{\alpha}\left[E_{\alpha}^{\psi}\left(\gamma, t, t_{0}\right)\right]=\gamma E_{\alpha}^{\psi}\left(\gamma, t, t_{0}\right)
$$

Now, the notion of general conformable exponential stability is introduced. 
Definition 12 The origin of system (1) is general conformable exponentially stable (GCES) if

$$
\|x\| \leq C\left\|x_{0}\right\| E_{\alpha}^{\psi}\left(-\gamma, t, t_{0}\right)
$$

with $t>t_{0}$ and $C, \gamma>0$.

Lemma 2 Let $g:\left[t_{0}, \infty\right) \rightarrow \mathbb{R}^{+}$be an $\alpha$-differentiable function on $\left(t_{0}, \infty\right)$ such that

$$
{ }^{\psi} D^{\alpha} g(t) \leq-\gamma g(t)
$$

where $\gamma>0$ and $\alpha \in(0,1)$. Then

$$
g(t) \leq g\left(t_{0}\right) E_{\alpha}^{\psi}\left(-\gamma, t, t_{0}\right)
$$

Proof Let $h(t)=g(t) E_{\alpha}^{\psi}\left(\gamma, t, t_{0}\right)$. Using the product rule, we have

$$
\begin{aligned}
{ }^{\psi} D^{\alpha} h(t) & =g(t) D^{\alpha}\left[E_{\alpha}^{\psi}\left(\gamma, t, t_{0}\right)\right]+{ }^{\psi} D^{\alpha}[g(t)] E_{\alpha}^{\psi}\left(\gamma, t, t_{0}\right) \\
& \leq \gamma g(t) E_{\alpha}^{\psi}\left(\gamma, t, t_{0}\right)-\gamma g(t) E_{\alpha}^{\psi}\left(\gamma, t, t_{0}\right) .
\end{aligned}
$$

Since ${ }^{\psi} D^{\alpha} h(t) \leq 0$, from Lemma $1 h(t)$ is a decreasing function. Hence, $h(t) \leq h\left(t_{0}\right)$, which gives the result.

The following theorems serve to prove stability in the sense of Lyapunov for the class of systems considered.

Theorem 2 Let $x=0$ be an equilibrium point for system (1). Let $V: \mathbb{R}^{+} \times \mathbb{R}^{n} \rightarrow R$ be an $\alpha$-differentiable function and $a_{i}(i=1,2,3)$ be arbitrary positive constants. If the following conditions are satisfied:

(i) $a_{1}\|x\|^{2} \leq V(t, x) \leq a_{2}\|x\|^{2}$,

(ii) ${ }^{\psi} D^{\alpha} V(t, x) \leq-a_{3}\|x\|^{2}$,

then the origin of system (1) is GCES.

Proof From conditions (i) and (ii) we have

$$
{ }^{\psi} D^{\alpha} V(t, x) \leq-\frac{a_{3}}{a_{2}} V(t, x) .
$$

Applying Lemma 2 to this inequality, we have

$$
V(t, x) \leq V\left(t_{0}, x_{0}\right) E_{\alpha}^{\psi}\left(-\frac{a_{3}}{a_{2}}, t, t_{0}\right) .
$$

Also from (i) we have

$$
\begin{aligned}
a_{1}\|x\|^{2} & \leq V\left(t_{0}, x_{0}\right) E_{\alpha}^{\psi}\left(-\frac{a_{3}}{a_{2}}, t, t_{0}\right) \\
& \leq a_{2} E_{\alpha}^{\psi}\left(-\frac{a_{3}}{a_{2}}, t, t_{0}\right)\left\|x_{0}\right\|^{2} .
\end{aligned}
$$


Thus

$$
\|x\|^{2} \leq\left(\frac{a_{2}}{a_{1}}\right) E_{\alpha}^{\psi}\left(-\frac{a_{3}}{a_{2}}, t, t_{0}\right)\left\|x_{0}\right\|^{2}
$$

and

$$
\|x\| \leq C\left\|x_{0}\right\| E_{\alpha}^{\psi}\left(-\gamma, t, t_{0}\right)
$$

with $C=\sqrt{a_{2} / a_{1}}$ and $\gamma=a_{3} / 2 a_{2}$. Therefore, the origin of system (1) is GCES.

Definition 13 A continuous function $k: \mathbb{R}^{+} \rightarrow \mathbb{R}^{+}$is said to belong to class $\mathcal{K}$ if it is strictly increasing and $k(0)=0$. It belongs to class $\mathcal{K}_{\infty}$ if $\lim _{t \rightarrow+\infty} k(t)=+\infty$.

Theorem 3 Let $x=0$ be an equilibrium point for system (1). Let $V: \mathbb{R}^{+} \times \mathbb{R}^{n} \rightarrow R$ be an $\alpha$-differentiable function and $k_{i}(i=1,2,3)$ be functions of class $\mathcal{K}$ satisfying

(i) $k_{1}(\|x\|) \leq V(t, x) \leq k_{2}(\|x\|)$

(ii) ${ }^{\psi} D^{\alpha} V(t, x) \leq-k_{3}(\|x\|)$,

then the origin of system (1) is GCES.

Furthermore, if $k_{i} \in \mathcal{K}_{\infty}(i=1,2,3)$, then the origin of system (1) is globally GCES.

Proof From condition (i), $V(t, x) \geq 0$; this means that $\lim _{t \rightarrow \infty} V(t, x)=L \geq 0$. However, from condition (ii) and Lemma $1, V(t, x)$ is a decreasing function, $\operatorname{sol}_{t \rightarrow \infty} V(t, x)=L=0$. To prove this, assume that $L>0$. From conditions (i) and (ii) and Lemma 1 , we have

$$
\begin{aligned}
{ }^{\psi} D^{\alpha} V(t, x) & \leq-k_{3} \circ k_{2}^{-1}(V(t, x)) \\
& \leq-k_{3} \circ k_{2}^{-1}(L) \\
& \leq-\lambda V\left(t_{0}, x_{0}\right)
\end{aligned}
$$

with $\lambda=\frac{k_{3} \circ k_{2}^{-1}(L)}{V\left(t_{0}, x_{0}\right)}$. Hence

$$
{ }^{\psi} D^{\alpha} V(t, x) \leq-\lambda V(t, x) .
$$

Thus from Lemma 2 we have

$$
V(t, x) \leq V\left(t_{0}, x_{0}\right) E_{\alpha}^{\psi}\left(-\lambda, t, t_{0}\right),
$$

which is a contradiction to the assumption for $L$. Therefore, $L=0$ and from (i), $\lim _{t \rightarrow \infty} x(t)=0$, so we have

$$
\|x\| \leq C\left\|x_{0}\right\| E_{\alpha}^{\psi}\left(-\gamma, t, t_{0}\right)
$$

for some $C, \gamma>0$. Hence, the origin of system (1) is GCES.

Considering the case where $k_{i} \in \mathcal{K}_{\infty}(i=1,2,3)$, (3) is satisfied $\forall x_{0} \in \mathbb{R}^{n}$. In this case, the origin of system (1) is globally GCES.

Remark 11 Note that the GCES concept implies exponential stability, taking $\alpha=1$, which leads to $\psi(t, \alpha)=1$. 


\section{Finite-time general conformable exponential stability}

From Remark 6, dynamics with GCD can be seen as an integer-order dynamics weighted by a time-dependent term

$$
{ }^{\psi} D^{\alpha} f=\psi(t, \alpha) \frac{d f(t)}{d t} .
$$

Thus, systems with GCD might be conceived as integer-order nonautonomous systems. Hence, the following definitions will be used [36].

Consider the integer order system

$$
\dot{x}=f(t, x), \quad t \in \mathbb{R}^{+}, x \in \mathbb{R}^{n},
$$

where $f: \mathbb{R}^{+} \times \mathbb{R}^{n} \rightarrow \mathbb{R}^{n}$ is a continuous function. $\phi_{t}^{x}(\tau)$ denotes a solution of (4) starting from $(t, x)$, and $\mathcal{S}(t, x)$ represents the set of all its solutions. $\mathcal{V}$ denotes a neighborhood of the origin in $\mathbb{R}^{n}$, and $\mathcal{B}_{\epsilon}$ is an open ball centered at the origin of radius $\epsilon$.

Definition 14 ([36]) A continuous function $f: \mathbb{R}^{+} \times \mathcal{V} \rightarrow \mathbb{R}$ is decrescent if there exists a $\mathcal{K}$ function $\psi$ such that

$$
|f(t, x)| \leq \psi(\|x\|) \quad \forall(t, x) \in \mathbb{R}^{+} \times \mathcal{V} .
$$

Definition 15 ([36]) A continuous function $f: \mathbb{R}^{+} \times \mathbb{R}^{n} \rightarrow \mathbb{R}$ is radially unbounded if there exists a $\mathcal{K}_{\infty}$ function $\varphi$ such that

$$
|f(t, x)| \geq \varphi(\|x\|) \quad \forall(t) \in \mathbb{R}^{+}, \forall x \in \mathbb{R}^{n} .
$$

Definition 16 ([36]) The origin of system (4) is weakly finite-time stable if

1. The origin is Lyapunov stable.

2. $\exists T\left(\phi_{t}^{x}\right) \in[0,+\infty)$ such that $\phi_{t}^{x}(\tau)=0, \forall \tau \geq t+T\left(\phi_{t}^{x}\right)$. Here, the term

$$
T_{0}\left(\phi_{t}^{x}\right)=\inf \left\{T\left(\phi_{t}^{x}\right) \geq 0: \phi_{t}^{x}(\tau)=0, \forall \tau \geq t+T\left(\phi_{t}^{x}\right)\right\}
$$

is called the settling time of the solution $\phi_{t}^{x}$.

3. Moreover, if $T_{0}(t, x)=\sup _{\phi_{t}^{x} \in \mathcal{S}(t, x)} T_{0}\left(\phi_{t}^{x}\right)<+\infty$, then the origin of system (4) is finite-time stable.

$T_{0}(t, x)$ is called the settling time with respect to the initial conditions of system (4).

Definition 17 ([36]) The origin of system (4) is uniformly finite-time stable if

1. The origin is uniformly asymptotically stable.

2. The origin is finite-time stable.

3. There exist a positive definite continuous function $\alpha: \mathbb{R}^{+} \rightarrow \mathbb{R}^{+}$such that the settling time with respect to the initial conditions of system (4) satisfies

$$
T_{0}(t, x) \leq \alpha(\|x\|)
$$


Now, consider again system (1)

$$
{ }^{\psi} D^{\alpha} x=f(t, x)
$$

Its equivalent integer-order form is

$$
\dot{x}=\frac{1}{\psi(t, \alpha)} f(t, x)=g(t, x)
$$

where the conformable function $\psi(t, \alpha)$ is different from zero $\forall t \in \mathbb{R}^{+}, \forall \alpha \in(0,1)$.

Proposition 1 Let the origin be an equilibrium point for system (1), wheref is continuous.

i) If there exists a continuously differentiable Lyapunov function $V(t, x)$ satisfying

$$
\dot{V}(t, x) \leq-r(V(t, x))
$$

with a positive definite continuous function $r: \mathbb{R}^{+} \rightarrow \mathbb{R}^{+}, r(0)=0$, such that for some $\epsilon>0$

$$
\int_{0}^{\epsilon} \frac{d z}{r(z)}<+\infty
$$

then the origin of system (1) is finite-time stable.

ii) If in addition $V(t, x)$ is decrescent, then the origin of system (1) is uniformly finite-time stable.

iii) If in addition system (1) is globally defined and $V(t, x)$ is radially unbounded, then the origin of system (1) is globally finite-time stable.

Proof Considering system (1) as its integer-order version (5), the proof follows from the proof of Proposition 4.1 in [36].

Definition 18 Let the origin be an equilibrium point for system (1), where $f$ is continuous. The origin is finite-time general conformable exponentially stable (FGCES) if

1. The origin is GCES;

2. The origin is finite-time stable.

If the origin is globally finite-time stable, then the origin of system (1) is globally FGCES.

Theorem 4 Let the origin be an equilibrium point for system (1), where $f$ is continuous. The origin is FGCES if there exists a continuously differentiable Lyapunov function $V(t, x)$ satisfying

(i) $k_{1}(\|x\|) \leq V(t, x) \leq k_{2}(\|x\|)$;

(ii) ${ }^{\psi} D^{\alpha} V(t, x) \leq-k_{3}(\|x\|)$, with $k_{i}(i=1,2,3)$ functions of class $\mathcal{K}$.

Furthermore, if $k_{1} \in \mathcal{K}_{\infty}$ and system (1) is globally defined, then the origin of system (1) is globally FGCES.

Proof From i and ii, the origin of system (1) is GCES (from Theorem 3), and thus there exists a continuous and differentiable Lyapunov function $V(t, x)$. 
Moreover, from the proof of Theorem 3, we get

$$
{ }^{\psi} D^{\alpha} V(t, x) \leq-\lambda V(t, x)
$$

and, following the alternative form of the GCD, we have

$$
\dot{V}(t, x) \leq-r(V(t, x))
$$

where $r(V(t, x))=\frac{\lambda V(t, x)}{\psi(t, \alpha)}$; it can be verified that $r(0)=0$. Furthermore, by using the change of variables $[0, V(t, x)] \rightarrow\left[t, t+T_{0}(t, x)\right]$ given by $z=V(\tau, x(\tau))$, we obtain

$$
\begin{aligned}
\int_{V(t, x)}^{0} \frac{d z}{-r(z)} & =\int_{t}^{t+T_{0}(t, x)} \frac{\psi(\tau, \alpha) \dot{V}(\tau, x)}{-\lambda V(\tau, x)} d \tau \\
& \leq \int_{t}^{t+T_{0}(t, x)} d \tau \\
& =T_{0}(t, x) .
\end{aligned}
$$

Finally, from [36] we have

$$
T_{0}(t, x)=\int_{0}^{V(t, x)} \frac{d z}{r(z)}<+\infty .
$$

Hence, the origin of system (1) is finite-time stable. Therefore, the origin of system (1) is FGCES.

Moreover, if $k_{1} \in \mathcal{K}_{\infty}$, it means that $V(t, x)$ is radially unbounded. If, in addition, system (1) is globally defined, the origin of system (1) is globally finite-time stable. Therefore, the origin of system (1) is globally FGCES.

\section{Design of nonlinear estimators}

In this section, the stability results presented previously are used to design a pair of estimators for dynamical systems with the GCD. Then it is proven that the estimation error obtained is FGCES.

Consider the following class of commensurate-order nonlinear systems with single output:

$$
\begin{aligned}
& { }^{\psi} D^{\alpha} \mathbf{x}=f(\mathbf{x}, \mathbf{u}), \quad \mathbf{x}(0)=\mathbf{x}_{0}, \\
& y=C \mathbf{x},
\end{aligned}
$$

where $\alpha \in(0,1), \mathbf{x} \in \mathbb{R}^{n}$ is the state vector, $\mathbf{u} \in \mathbb{R}^{m}$ is the control input, $y \in \mathbb{R}$ is the output, and $f: \mathbb{R}^{n} \times \mathbb{R}^{m} \rightarrow \mathbb{R}^{n}$ is a locally Lipschitz vector function in $\mathbf{x}$ and uniformly bounded in $\mathbf{u}$.

Rewrite the system to its canonical form

$$
\begin{aligned}
& { }^{\psi} D^{\alpha} \mathbf{x}=A \mathbf{x}+\Upsilon(\mathbf{x}, \mathbf{u}), \quad \mathbf{x}(0)=\mathbf{x}_{0}, \\
& y=C \mathbf{x},
\end{aligned}
$$


where $A$ is an upper shift matrix $\left(A: \mathbb{R}^{n} \rightarrow \mathbb{R}^{n}, A_{i, j}=\delta_{i+1, j}\right.$, with $\delta_{i, j}$ the Kronecker delta), $C=\left[\begin{array}{llll}1 & 0 & 0 & \ldots\end{array}\right]$, the pair $(A, C)$ is observable, and $\Upsilon(\mathbf{x}, \mathbf{u})$ is a nonlinear vector that satisfies the Lipschitz condition

$$
\|\Upsilon(\mathbf{x}, \mathbf{u})-\Upsilon(\hat{\mathbf{x}}, \mathbf{u})\| \leq \varphi\|\mathbf{x}-\hat{\mathbf{x}}\|
$$

locally in a region $D$. The estimators will be designed based on this equivalent system; their estimation error is bounded by the general conformable exponential function (2).

\subsection{LQR-based estimator}

Consider the following estimator based on a linear quadratic regulator (LQR) [50]:

$$
{ }^{\psi} D^{\alpha} \mathbf{x}=A \hat{\mathbf{x}}+\Upsilon(\hat{\mathbf{x}}, \mathbf{u})+\sum_{i=1}^{m} \mathbf{K}_{i}(y-C \hat{\mathbf{x}})^{2 i-1},
$$

where $\hat{\mathbf{x}}, \mathbf{K}_{i} \in \mathbb{R}^{n}, 1 \leq i \leq m$. Consider the following lemma.

Lemma 3 ([50]) Given a stable $n \times m$ matrix $\hat{A}$ and $\gamma>0$, there exists a positive definite, symmetric matrix $P$ such that

$$
\hat{A}^{T} P+P \hat{A}+\gamma^{2} P P+I<0
$$

if and only if there exists another positive definite, symmetric matrix $\hat{P}$ such that

$$
\hat{A} \hat{P}+\hat{P} \hat{A}^{T}+\gamma^{2} \hat{P} \hat{P}+I<0
$$

The following LMI is equivalent to (10)

$$
\left[\begin{array}{cc}
-\hat{A}^{T} P-P \hat{A}-I & \gamma P \\
\gamma P & I
\end{array}\right]>0 .
$$

Now, for some $\varepsilon>0$,

$$
\hat{A}^{T} P+P \hat{A}+\gamma^{2} P P+I+\varepsilon I=0 .
$$

Let $v=\Upsilon(\mathbf{x}, \mathbf{u})-\Upsilon(\hat{\mathbf{x}}, \mathbf{u})$. Considering condition (8), the estimation error $\mathbf{e}=\mathbf{x}-\hat{\mathbf{x}}$, and the solution $P$ of (11), we have

$$
2 \mathbf{e}^{T} P v \leq \varphi^{2} \mathbf{e}^{T} P P \mathbf{e}+\mathbf{e}^{T} \mathbf{e} .
$$

Remark 12 An estimator is said to be finite-time general conformable exponentially stable if the estimation error $\mathbf{e}$ obtained with it is FGCES.

Theorem 5 Consider system (7) with pair $(A, C)$ observable. If $\bar{A}=A-\mathbf{K}_{1} C$ is stable and $M_{i}=P^{i-1} \mathbf{K}_{i} C$ is positive semidefinite for $2 \leq i \leq m$, then the LQR-based estimator (9) is a finite-time general conformable exponentially stable estimator for system (6). 
Proof From (7) and (9), the dynamics of $\mathbf{e}$ is

$$
{ }^{\psi} D^{\alpha} \mathbf{e}=\bar{A} \mathbf{e}+F-\sum_{i=2}^{m} \mathbf{K}_{i}(C \mathbf{e})^{2 i-1} .
$$

As $\bar{A}$ is stable and $\varphi>0$, from Lemma $3 \exists P>0$. Consider $V=\|\mathbf{e}\|_{P}^{2}=\mathbf{e}^{T} P \mathbf{e}$ a candidate Lyapunov function that satisfies the Rayleigh-Ritz inequality

$$
\lambda_{\text {min }}(P)\|\mathbf{e}\|^{2} \leq V \leq \lambda_{\max }(P)\|\mathbf{e}\|^{2} .
$$

From Remark 9, (12), and (13) we have

$$
\begin{aligned}
{ }^{\psi} D^{\alpha} V & =2 \mathbf{e}^{T} P^{\psi} D^{\alpha} \mathbf{e} \\
& =2 \mathbf{e}^{T} P\left[\bar{A} \mathbf{e}+v-\sum_{i=2}^{m} \mathbf{K}_{i}(C \mathbf{e})^{2 i-1}\right] \\
& \leq \mathbf{e}^{T}\left[\hat{A}^{T} P+P \hat{A}+\gamma^{2} P P+I\right] \mathbf{e}-2 \sum_{i=2}^{m}(C \mathbf{e})^{2 i-2} \mathbf{e}^{T} P \mathbf{K}_{i} C \mathbf{e} \\
& =-\varepsilon\|\mathbf{e}\|^{2}-\sum_{i=2}^{m}(C \mathbf{e})^{2 i-2} \mathbf{e}^{T}\left(P^{2-i} M_{i}\right) \mathbf{e} .
\end{aligned}
$$

Given that $M_{i}=P^{i-1} \mathbf{K}_{i} C \geq 0$, we have

$$
{ }^{\psi} D^{\alpha} V \leq-\varepsilon\|\mathbf{e}\|^{2}
$$

From Theorem 2, (15), and (16) it follows that the origin of system (14) is GCES, and we have

$$
\|\mathbf{e}\| \leq C\left\|\mathbf{e}_{0}\right\| E_{\alpha}^{\psi}\left(-\gamma, t, t_{0}\right)
$$

with $C=\sqrt{\frac{\lambda_{\max }(P)}{\lambda_{\min }(P)}}, \gamma=\frac{\varepsilon}{2 \lambda_{\max }(P)}$ and $\mathbf{e}_{0}=\mathbf{e}\left(t_{0}\right)$.

Furthermore, from (16) and the properties of norms we have

$$
\begin{aligned}
&{ }^{\psi} D^{\alpha} V \leq-\varepsilon\|\mathbf{e}\|^{2}, \\
& \dot{V} \leq-\frac{\varepsilon}{\psi(t, \alpha)}\|\mathbf{e}\|^{2} \\
& \leq-\frac{c_{1} \varepsilon}{\psi(t, \alpha)}\|\mathbf{e}\|_{P}^{2} \\
&=-r(V),
\end{aligned}
$$

where $r(V)=\frac{c_{1} \varepsilon V(t, x)}{\psi(t, \alpha)}$. We have that $r(0)=0$ and

$$
\begin{aligned}
\int_{V(t, x)}^{0} \frac{d z}{-r(z)} & =\int_{t}^{t+T_{0}(t, x)} \frac{\psi(\tau, \alpha) \dot{V}(\tau, x)}{-c_{1} \varepsilon V(\tau, x)} d \tau \\
& \leq \int_{t}^{t+T_{0}(t, x)} d \tau
\end{aligned}
$$




$$
=T_{0}(t, x)<+\infty
$$

thus, the origin of system (14) is finite-time stable.

Therefore, the origin of system (14) is FGCES, and hence system (9) is a finite-time general conformable exponentially stable estimator for system (6).

\subsection{High-gain observer}

Consider the following high-gain observer (HGO) [51]:

$$
{ }^{\psi} D^{\alpha} \hat{\mathbf{x}}=A \hat{\mathbf{x}}+\Upsilon(\hat{\mathbf{x}}, \mathbf{u})+F_{\infty}^{-1} C^{T}(y-C \hat{\mathbf{x}}),
$$

where $F_{\infty}=\lim _{t \rightarrow \infty} F_{\theta}(t)$, with $F_{\theta}(t)$ positive definite solution of

$$
{ }^{\psi} D^{\alpha} F_{\theta}(t)=-\theta F_{\theta}(t)-A^{T} F_{\theta}(t)-F_{\theta}(t) A+C^{T} C, \quad F_{0}=F_{\theta}(0)
$$

Remark 13 Given that $F_{\infty}$ is constant, ${ }^{\psi} D^{\alpha} F_{\infty}=0$, and hence it may be calculated from

$$
-\theta F_{\infty}-A^{T} F_{\infty}-F_{\infty} A+C^{T} C=0 .
$$

The coefficients of $F_{\infty}$ are given by

$$
\left(F_{\infty}\right)_{i, j}=\frac{\alpha_{i, j}}{\theta^{i+j-1}}
$$

where $\alpha_{i, j}$ is symmetric positive definite, independent of $\theta$.

Theorem 6 The high-gain observer (17) is a finite-time general conformable exponentially stable estimator for system (6).

Proof From (7) and (17), the dynamics of $\mathbf{e}$ is

$$
{ }^{\psi} D^{\alpha} \mathbf{e}=\left(A+F_{\infty}^{-1} C^{T} C\right) \mathbf{e}+v
$$

with $v=\Upsilon(\mathbf{x}, \mathbf{u})-\Upsilon(\hat{\mathbf{x}}, \mathbf{u})$. Consider $V=\|\mathbf{e}\|_{F_{\infty}}^{2}=\mathbf{e}^{T} F_{\infty} \mathbf{e}$ a candidate Lyapunov function that satisfies the Rayleigh-Ritz inequality

$$
\lambda_{\text {min }}\left(F_{\infty}\right)\|\mathbf{e}\|^{2} \leq V \leq \lambda_{\text {max }}\left(F_{\infty}\right)\|\mathbf{e}\|^{2} .
$$

Moreover, from Remark 9 and (18) we have

$$
\begin{aligned}
{ }^{\psi} D^{\alpha} V & =2 \mathbf{e}^{T} F_{\infty}{ }^{\psi} D^{\alpha} \mathbf{e} \\
& =2 \mathbf{e}^{T} F_{\infty}\left[\left(A+F_{\infty}^{-1} C^{T} C\right) \mathbf{e}+v\right] \\
& \leq-\theta \mathbf{e}^{T} F_{\infty} \mathbf{e}+2 \mathbf{e}^{T} F_{\infty} v .
\end{aligned}
$$

Thus, from condition (8), for $\theta>2 \varphi+1$,

$$
{ }^{\psi} D^{\alpha} V \leq-(\theta-2 \varphi-1)\left\|F_{\infty}\right\|\|\mathbf{e}\|^{2} .
$$


According to Theorem 2, from (20) and (21) it follows the origin of system (19) is GCES, and we have

$$
\|\mathbf{e}\| \leq C\left\|\mathbf{e}_{0}\right\| E_{\alpha}^{\psi}\left(-\gamma, t, t_{0}\right)
$$

with $C=\sqrt{\frac{\lambda_{\max }\left(F_{\infty}\right)}{\lambda_{\min }\left(F_{\infty}\right)}}, \gamma=\frac{(\theta-2 \varphi-1)\left\|F_{\infty}\right\|}{2 \lambda_{\max }\left(F_{\infty}\right)}$, and $\mathbf{e}_{0}=\mathbf{e}\left(t_{0}\right)$.

Furthermore, from (21) and the properties of norms, we have

$$
\begin{aligned}
&{ }^{\psi} D^{\alpha} V \leq-(\theta-2 \varphi-1)\left\|F_{\infty}\right\|\|\mathbf{e}\|^{2} \\
& \dot{V} \leq-\frac{\theta-2 \varphi-1}{\psi(t, \alpha)}\|\mathbf{e}\|^{2} \\
& \leq-\frac{c_{1}(\theta-2 \varphi-1)}{\psi(t, \alpha)}\|\mathbf{e}\|_{F_{\infty}}^{2} \\
&=-r(V)
\end{aligned}
$$

where $r(V)=\frac{c_{1}(\theta-2 \varphi-1) V(t, x)}{\psi(t, \alpha)}$. We have that $r(0)=0$ and

$$
\begin{aligned}
\int_{V(t, x)}^{0} \frac{d z}{-r(z)} & =\int_{t}^{t+T_{0}(t, x)} \frac{\psi(\tau, \alpha) \dot{V}(\tau, x)}{-c_{1}(\theta-2 \varphi-1) V(\tau, x)} d \tau \\
& \leq \int_{t}^{t+T_{0}(t, x)} d \tau \\
& =T_{0}(t, x)<+\infty .
\end{aligned}
$$

Thus, the origin of system (19) is finite-time stable.

Therefore, the origin of system (19) is FGCES, and hence system (17) is a finite-time general conformable exponentially stable estimator for system (6).

\section{Numerical simulations}

In this section, the results of numerical simulations of the estimation scheme proposed are presented. For this, the general conformable estimators were designed and applied based on the models of two dynamical systems with GCD. In addition, the performance of the scheme with integer, fractional, and general conformable dynamics is compared. The simulations were carried out using Simulink ${ }^{\circ}$ from MATLAB $^{\circ}$; moreover, to implement the fractional case, the noninteger fractional derivative from D. Valério was used [52].

\subsection{Simple pendulum}

Consider the extension of the simple pendulum to its noninteger order version, studied e.g. in [53-55]:

$$
\begin{aligned}
& { }^{\psi} D^{\alpha} x_{1}=x_{2}, \\
& { }^{\psi} D^{\alpha} x_{2}=-\frac{g}{L} \sin \left(x_{1}\right), \\
& y=x_{1},
\end{aligned}
$$


where $x_{1}=\theta$ (angular position), $x_{2}=\omega$ (angular velocity), $g=9.81 \mathrm{~m} / \mathrm{s}^{2}$, and $L=1 \mathrm{~m}$. Rewriting it to the canonical form, we have

$$
\begin{aligned}
& { }^{\psi} D^{\alpha} \mathbf{x}=A \mathbf{x}+\Upsilon(\mathbf{x}), \quad x(0)=x_{0}, \\
& y=C \mathbf{x}
\end{aligned}
$$

with $A=\left[\begin{array}{ll}0 & 1 \\ 0 & 0\end{array}\right], \Upsilon(\mathbf{x})=\left[\begin{array}{c}0 \\ -\frac{g}{L} \sin \left(x_{1}\right)\end{array}\right]$, Lipschitz constant $\varphi=\frac{g}{L}$, and $C=\left[\begin{array}{ll}1 & 0\end{array}\right]$.

A mechanical system with noninteger dynamics has the effect of additional damping on the trajectories $[54,55]$ which can be understood as an extra parameter that varies with the choice of the order. When using the GCD, due to the freedom to choose the order $\alpha$ as well as the conformable function $\psi(t, \alpha)$, the resulting system gets some additional time-varying parameters. For instance, the graphs of the states show that the oscillation frequency varies.

From (9) with $m=3$, the LQR-based estimator for system (22) is

$$
\begin{aligned}
& { }^{\psi} D^{\alpha} \hat{\mathbf{x}}=A \hat{\mathbf{x}}+\Upsilon(\hat{\mathbf{x}})+\mathbf{K}_{1} C(\mathbf{x}-\hat{\mathbf{x}})+\mathbf{K}_{2}[C(\mathbf{x}-\hat{\mathbf{x}})]^{3}+\mathbf{K}_{3}[C(\mathbf{x}-\hat{\mathbf{x}})]^{5}, \\
& y=\hat{\mathbf{x}},
\end{aligned}
$$

where $\mathbf{K}_{i}=\left[K_{i 1} K_{i 2}\right]^{T}$.

For these simulations $\alpha=0.98$, the gain vectors were selected as $\mathbf{K}_{1}=[4.00763 .1305]^{T}$, $\mathbf{K}_{2}=\left[\begin{array}{l}4.9054 .905\end{array}\right]^{T}, \mathbf{K}_{3}=\left[\begin{array}{ll}5 & 2.4525\end{array}\right]^{T}$, and the initial conditions are $x_{1}(0)=\pi / 2, x_{2}(0)=0$, $\hat{x}_{1}(0)=\pi, \hat{x}_{2}(0)=0$. For implementing the GCD, the function $\psi(t, \alpha)=0.99 t^{30(1-\alpha)}+0.01$ has been chosen.

Figures 1 and 2 show the state estimations; it can be seen that the estimated signals reach the equilibrium before 1 second. Figures 3 and 4 show the comparison of the estimation errors obtained from the integer, fractional, and GCD operators for the same models and parameters in logarithmic time, while Figs. 5 and 6 show in major detail these comparisons. Finally, Figs. 7 and 8 show the performance measure of the errors, obtained using the integral of the square of the error (ISE)

$$
I S E=\int_{0}^{T} e^{2}(t) d t
$$

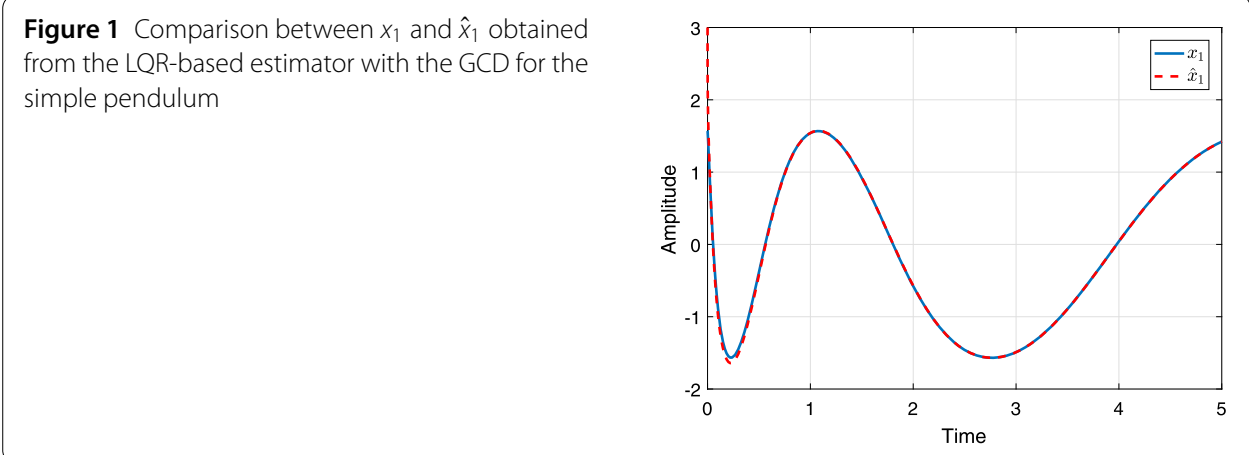


Figure 2 Comparison between $x_{2}$ and $\hat{x}_{2}$ obtained from the LQR-based estimator with the GCD for the simple pendulum

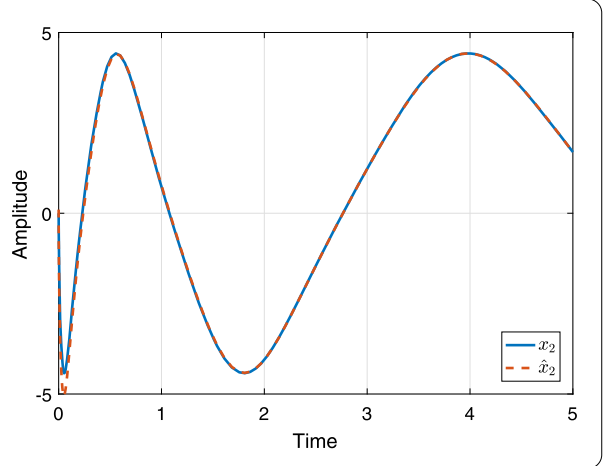

Figure 3 Comparison between $e_{1}$ obtained from the LQR-based estimator with integer, fractional, and GCD for the simple pendulum

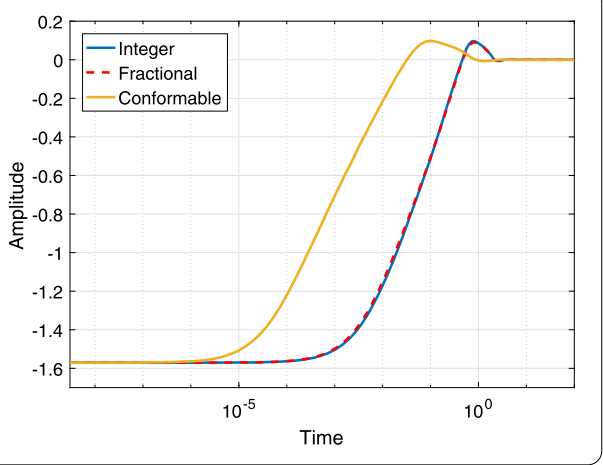

Figure 4 Comparison between $e_{2}$ obtained from the LQR-based estimator with integer, fractional, and GCD for the simple pendulum

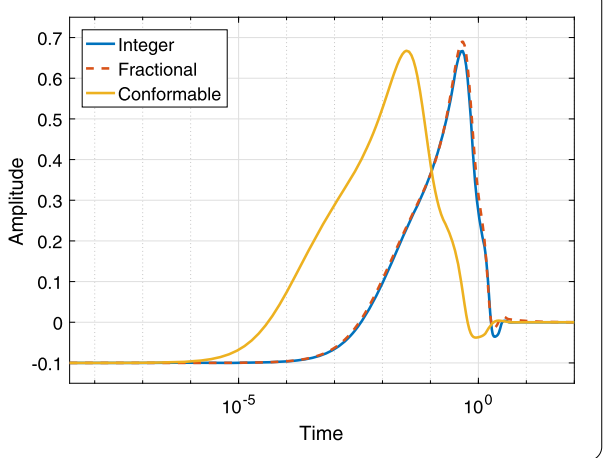

From these results it can be seen that the fractional case performs slightly better than the integer one, but it does not reach the equilibrium in the time scale shown (corresponding to $100 \mathrm{~s}$ ); this can be appreciated also in a slightly increasing slope in the ISE for the fractional case. Moreover, the other cases present finite-time stabilization, but with the GCD operator, the signals converge faster and smoother.

Consider now HGO (17) for the pendulum system

$$
\begin{aligned}
& { }^{\psi} D^{\alpha} \hat{x}_{1}=\hat{x}_{2}+2 \theta\left(x_{1}-\hat{x}_{1}\right), \\
& { }^{\psi} D^{\alpha} \hat{x}_{2}=-\frac{g}{L} \sin \left(\hat{x}_{1}\right)+\theta^{2}\left(x_{1}-\hat{x}_{1}\right),
\end{aligned}
$$$$
y=\hat{\mathbf{x}} .
$$ 
Figure 5 Details of comparison between $e_{1}$ obtained from the LQR-based estimator with integer, fractional, and GCD for the simple pendulum

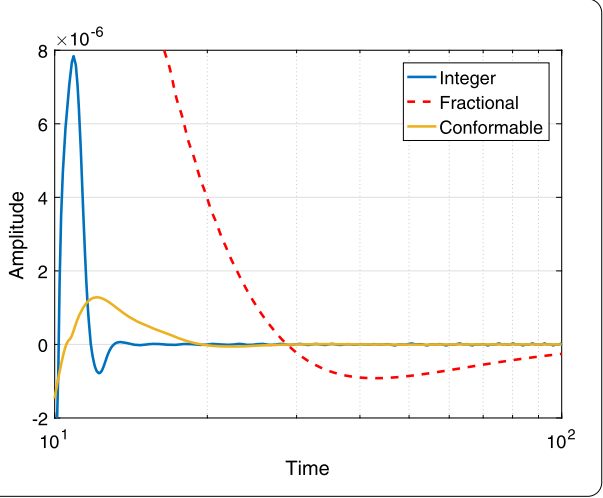

Figure 6 Details of comparison between $e_{2}$ obtained from the LQR-based estimator with integer, fractional, and GCD for the simple pendulum

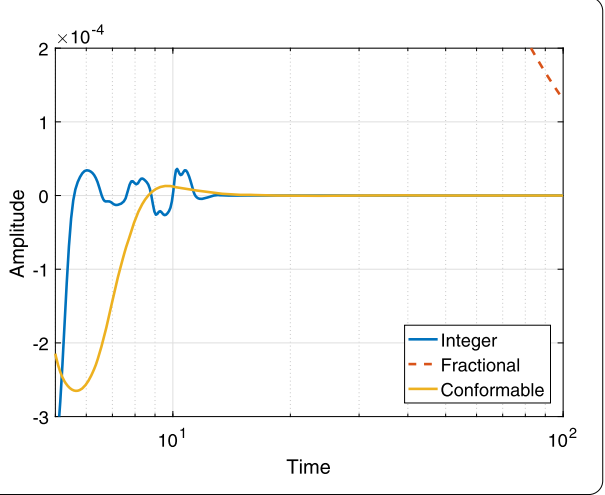

Figure 7 Comparison between the ISE for $e_{1}$ obtained from the LQR-based estimator with integer, fractional, and GCD for the simple pendulum

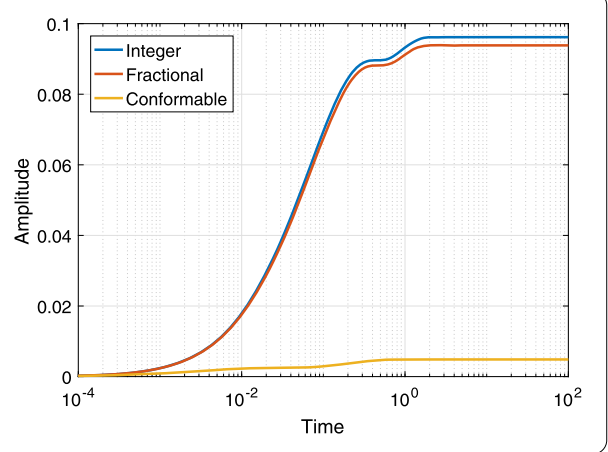

Simulations were performed using the same initial conditions of the former estimator with $\theta=25$ and the same conformable function. Figures 9 and 10 show the state estimations; it can be seen that the estimated signals reach the equilibrium around 0.25 seconds. Figures 11 and 12 show the comparison of the estimation errors obtained from the integer, fractional, and GCD operators for the same models and parameters in logarithmic time, while Figs. 13 and 14 show in major detail these comparisons. Finally, Figs. 15 and 16 show the performance measure of the errors, obtained using the ISE.

Similar to the results obtained from the LQR-estimator, it can be seen that the fractional case performs slightly better than the integer one; however, it does not reach the equilibrium in the time scale shown (corresponding to $100 \mathrm{~s}$ ); a major amplification would show that the signals still fail to reach the origin. Also, a slightly increasing slope appears in the 
Figure 8 Comparison between the ISE for $e_{2}$ obtained from the LQR-based estimator with integer, fractional, and GCD for the simple pendulum

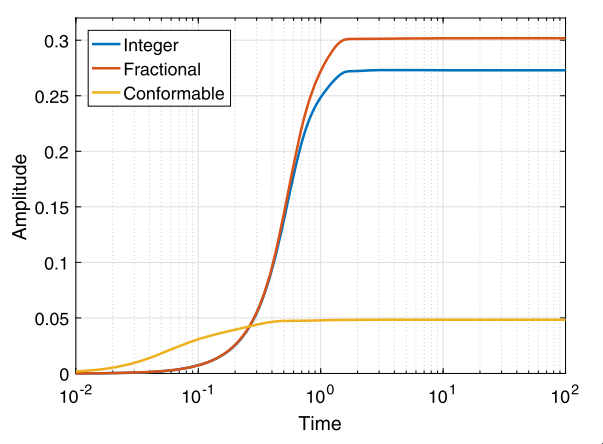

Figure 9 Comparison between $x_{1}$ and $\hat{x}_{1}$ obtained from the high-gain observer with the GCD for the simple pendulum

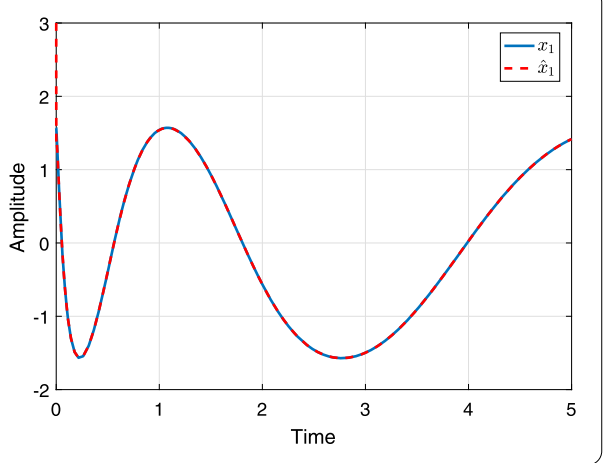

Figure 10 Comparison between $x_{2}$ and $\hat{x}_{2}$ obtained from the high-gain observer with the GCD for the simple pendulum

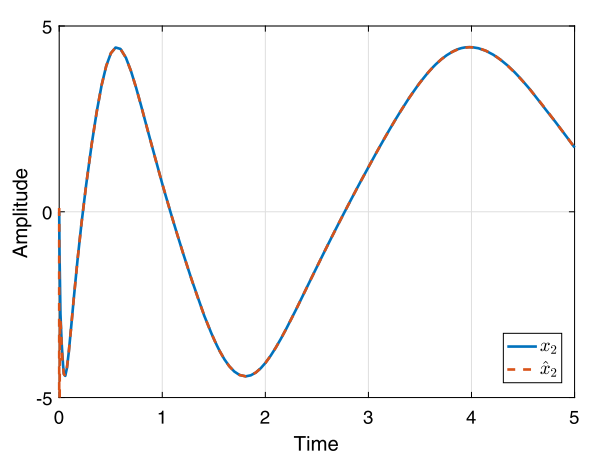

Figure 11 Comparison between $e_{1}$ obtained from the high-gain observer with integer, fractional, and GCD for the simple pendulum

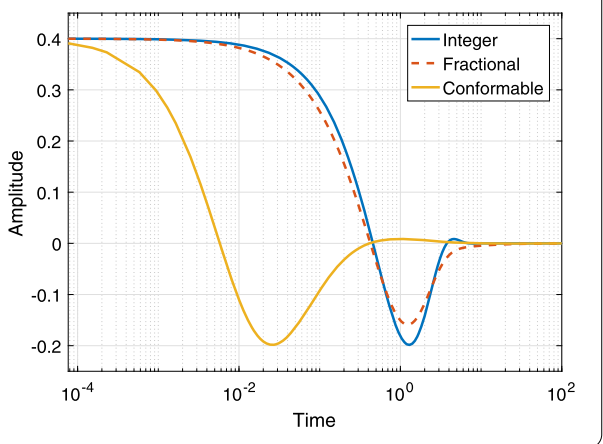

ISE for the fractional case. Furthermore, the other cases present finite-time stabilization, but with the GCD operator, the signals converge faster and smoother. 
Figure 12 Comparison between $e_{2}$ obtained from the high-gain observer with integer, fractional, and GCD for the simple pendulum

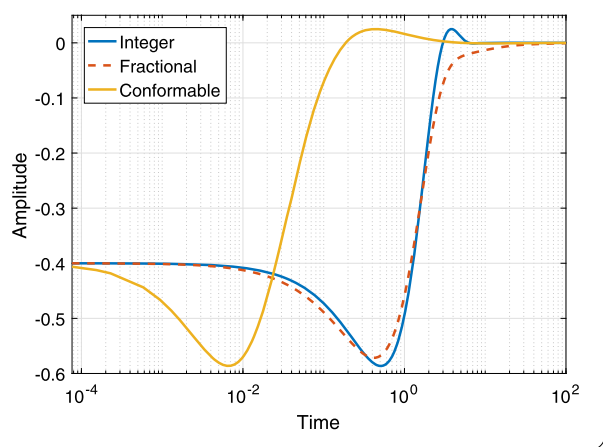

Figure 13 Details of comparison between $e_{1}$ obtained from the high-gain observer with integer, fractional, and GCD for the simple pendulum

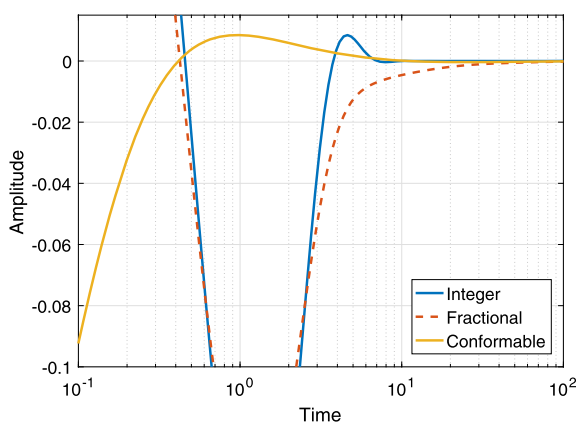

Figure 14 Details of comparison between $e_{2}$ obtained from the high-gain observer with integer, fractional, and GCD for the simple pendulum

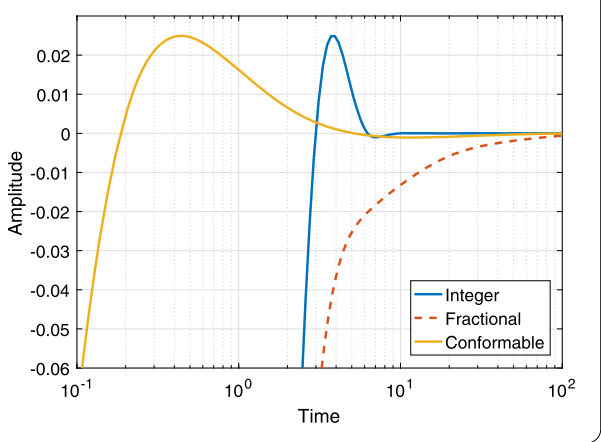

Figure 15 Comparison between the ISE for $e_{1}$ obtained from the high-gain observer with integer, fractional, and GCD for the simple pendulum

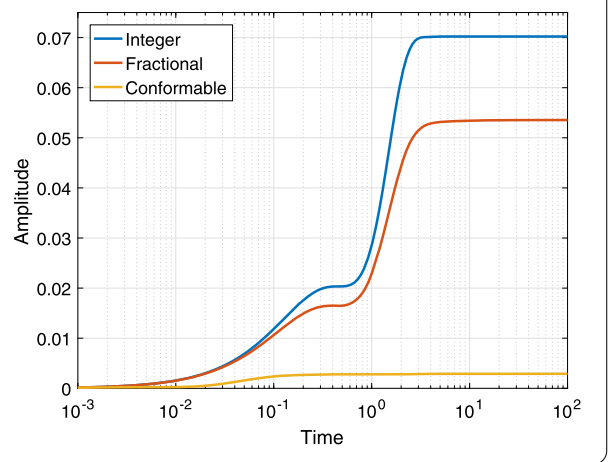

\subsection{Van der Pol oscillator}

The Van der Pol oscillator is a chaotic system proposed to study oscillations in vacuum tube circuits [56]. Its noninteger counterpart has been studied e.g. in [57-59]. The ex- 
Figure 16 Comparison between the ISE for $e_{2}$ obtained from the high-gain observer with integer, fractional, and GCD for the simple pendulum

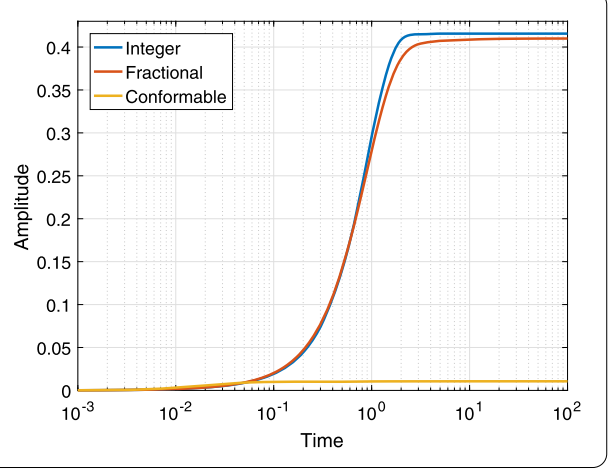

tended model is

$$
\begin{aligned}
& { }^{\psi} D^{\alpha} x_{1}=x_{2}, \\
& { }^{\psi} D^{\alpha} x_{2}=-x_{1}-\varepsilon\left(x_{1}^{2}-1\right) x_{2}, \\
& y=x_{1},
\end{aligned}
$$

where $\varepsilon$ is a control parameter. Rewriting it to its canonical form, we have

$$
\begin{aligned}
& { }^{\psi} D^{\alpha} \mathbf{x}=A \mathbf{x}+\Upsilon(\mathbf{x}), \quad x(0)=x_{0}, \\
& y=C \mathbf{x}
\end{aligned}
$$

with $A=\left[\begin{array}{ll}0 & 1 \\ 0 & 0\end{array}\right], \Upsilon(\mathbf{x})=\left[\begin{array}{c}0 \\ -x_{1}-\varepsilon\left(x_{1}^{2}-1\right) x_{2}\end{array}\right]$, Lipschitz constant $\varphi=\max \left\{1+2 \varepsilon\left|x_{1}\right|\left|x_{2}\right|+\varepsilon+\varepsilon\left|x_{1}^{2}\right|\right\}$, and $C=\left[\begin{array}{ll}1 & 0\end{array}\right]$.

This case may be seen as a master-slave synchronization problem. The Van der Pol system will perform as the master system, and the estimators will serve as the slaves, so they will look for synchronizing with it.

From (9) with $m=3$, the LQR-based estimator for system (23) is

$$
\begin{aligned}
& { }^{\psi} D^{\alpha} \hat{\mathbf{x}}=A \hat{\mathbf{x}}+\Upsilon(\hat{\mathbf{x}})+\mathbf{K}_{1} C(\mathbf{x}-\hat{\mathbf{x}})+\mathbf{K}_{2}[C(\mathbf{x}-\hat{\mathbf{x}})]^{3}+\mathbf{K}_{3}[C(\mathbf{x}-\hat{\mathbf{x}})]^{5}, \\
& y=\hat{\mathbf{x}},
\end{aligned}
$$

where $\mathbf{K}_{i}=\left[K_{i 1} K_{i 2}\right]^{T}$.

The gain vectors have been chosen as $\mathbf{K}_{1}=\left[\begin{array}{lll}2.3094 & 1.5166\end{array}\right]^{T}, \mathbf{K}_{2}=\left[\begin{array}{ll}1.15 & 1.15\end{array}\right]^{T}, \mathbf{K}_{3}=$ [3 0.575$]^{T}$. The parameters of the system are $\alpha=0.9, \varepsilon=0.1$ and the initial conditions have been chosen as $x_{1}(0)=-0.25, x_{2}(0)=1.2, \hat{x}_{1}(0)=3, \hat{x}_{2}(0)=1.8$. With these values, the Lipschitz constant is set as $\varphi=2.3$. For implementing the GCD, the function $\psi(t, \alpha)=$ $0.99 t^{10(1-\alpha)}+0.01$ has been chosen.

Figures 17 and 18 show the state estimations; it can be seen that the estimated signals reach the equilibrium around 2.5 seconds. Figures 19 and 20 show the comparison of the estimation errors obtained from the integer, fractional, and GCD operators for the same models and parameters in logarithmic time, while Figs. 21 and 22 show in major detail these comparisons. Finally, Figs. 23 and 24 show the performance measure of the errors obtained using the ISE. 
Figure 17 Comparison between $x_{1}$ and $\hat{x}_{1}$ obtained from the LQR-based estimator with the GCD for the Van der Pol oscillator

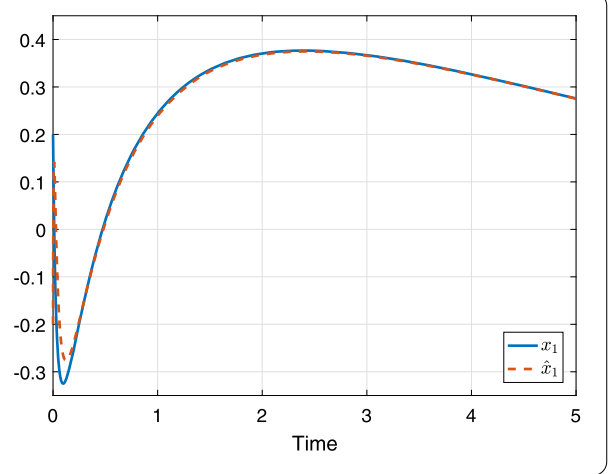

Figure 18 Comparison between $x_{2}$ and $\hat{x}_{2}$ obtained from the LQR-based estimator with the GCD for the Van der Pol oscillator

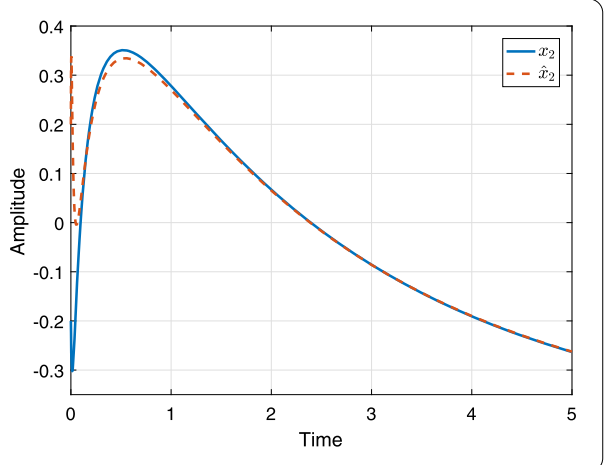

Figure 19 Comparison between $e_{1}$ obtained from the LQR-based estimator with integer, fractional, and GCD for the Van der Pol oscillator

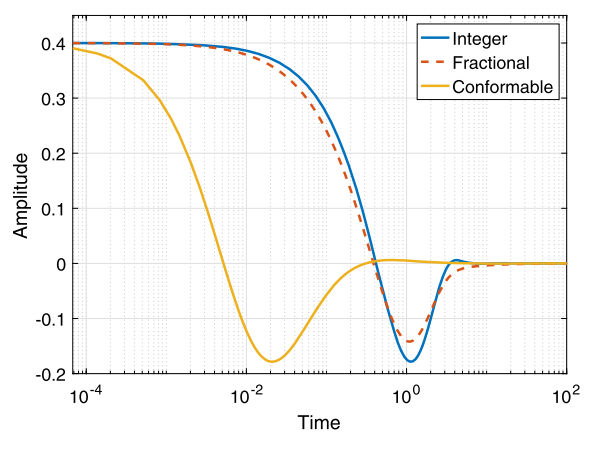

Figure 20 Comparison between $e_{2}$ obtained from the LQR-based estimator with integer, fractional, and GCD for the Van der Pol oscillator

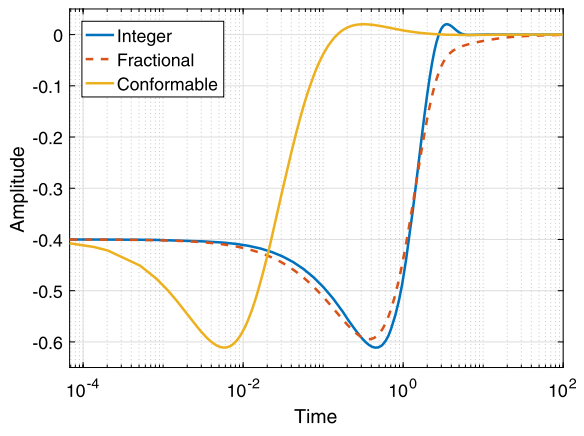


Figure 21 Details of comparison between $e_{1}$ obtained from the LQR-based estimator with integer, fractional, and GCD for the Van der Pol oscillator

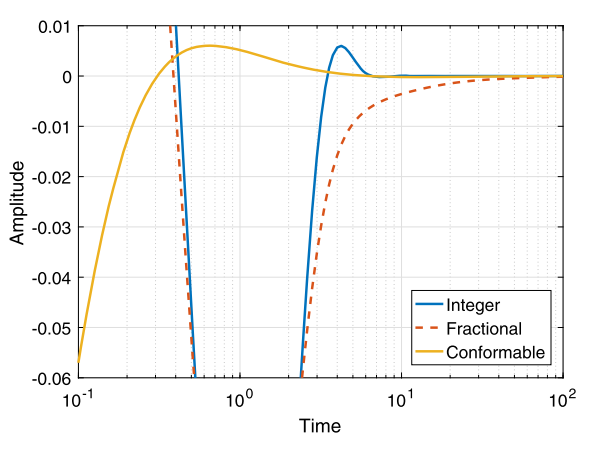

Figure 22 Details of comparison between $e_{2}$ obtained from the LQR-based estimator with integer, fractional, and GCD for the Van der Pol oscillator

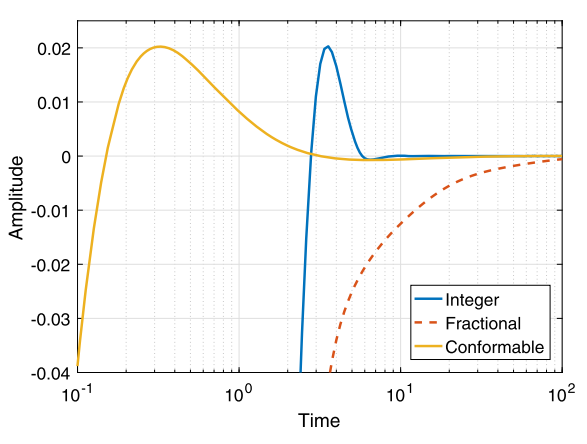

Figure 23 Comparison between the ISE for $e_{1}$ obtained from the LQR-based estimator with integer, fractional, and GCD for the Van der Pol oscillator

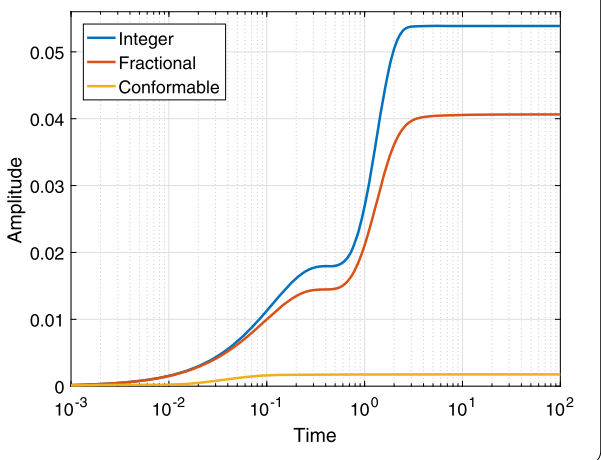

Figure 24 Comparison between the ISE for $e_{2}$ obtained from the LQR-based estimator with integer, fractional, and GCD for the Van der Pol oscillator

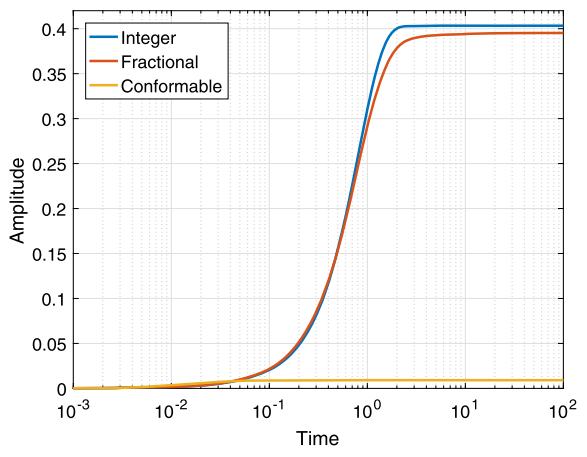


Figure 25 Phase portrait of the Van der Pol oscillator with the estimated states obtained from the LQR-based estimator

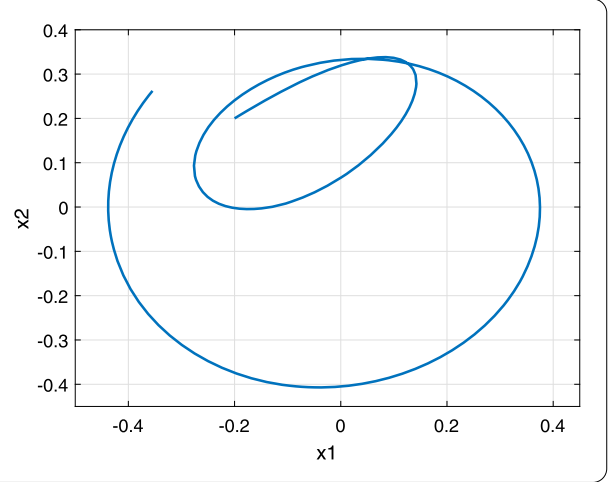

Figure 26 Comparison between $x_{1}$ and $\hat{x}_{1}$ obtained from the high-gain observer with the GCD for the Van der Pol oscillator

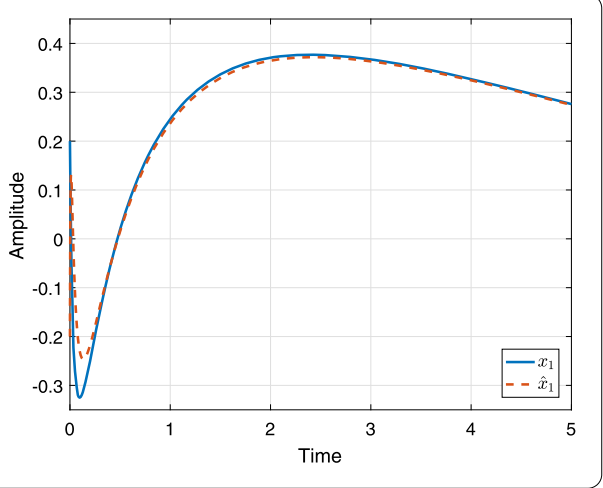

From these results it can be seen that the fractional case performs better than the other, with a smaller overshoot, but it does not reach the equilibrium in the time scale shown (corresponding to $100 \mathrm{~s}$ ); this can be appreciated also in a slightly increasing slope in the ISE for the fractional case. Moreover, the other cases present finite-time stabilization, but with the GCD operator, the signals converge faster and smoother. Furthermore, Fig. 25 shows the phase portrait obtained with $\hat{x}_{1}$ and $\hat{x}_{2}$.

Now, consider HGO (17). For the Van der Pol oscillator, the observer is

$$
\begin{aligned}
& { }^{\psi} D^{\alpha} \hat{x}_{1}=\hat{x}_{2}+2 \theta\left(x_{1}-\hat{x}_{1}\right), \\
& { }^{\psi} D^{\alpha} \hat{x}_{2}=-\hat{x}_{1}-\varepsilon\left(\hat{x}_{1}^{2}-1\right) \hat{x}_{2}+\theta^{2}\left(x_{1}-\hat{x}_{1}\right), \\
& y=\hat{\mathbf{x}} .
\end{aligned}
$$

Simulations were performed using the same initial conditions of the former estimator with $\theta=1$ and the same conformable function. Figures 26 and 27 show the state estimations; it can be seen that the estimated signals reach the equilibrium around 2.5 seconds. Figures 28 and 29 show the comparison of the estimation errors obtained from the integer, fractional, and GCD operators for the same models and parameters in logarithmic time, while Figs. 30 and 31 show in detail these comparisons. Finally, Figs. 32 and 33 show the performance measure of the errors, which was obtained using the ISE.

Similar to the results obtained from the LQR-estimator, it can be seen that the fractional case performs better than the other, with a smaller overshoot, but it does not reach the equilibrium in the time scale shown (corresponding to $100 \mathrm{~s}$ ); this can be appreciated in a slightly increasing slope in the ISE for the fractional case. Furthermore, the other cases 
Figure 27 Comparison between $x_{2}$ and $\hat{x}_{2}$ obtained from the high-gain observer with the GCD for the Van der Pol oscillator

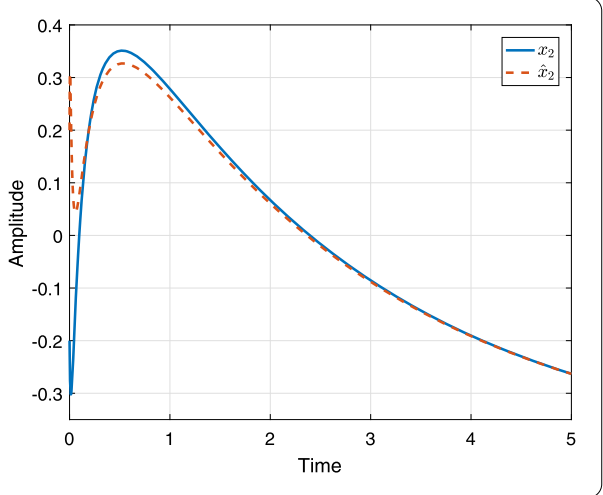

Figure 28 Comparison between $e_{1}$ obtained from the high-gain observer with integer, fractional, and GCD for the Van der Pol oscillator

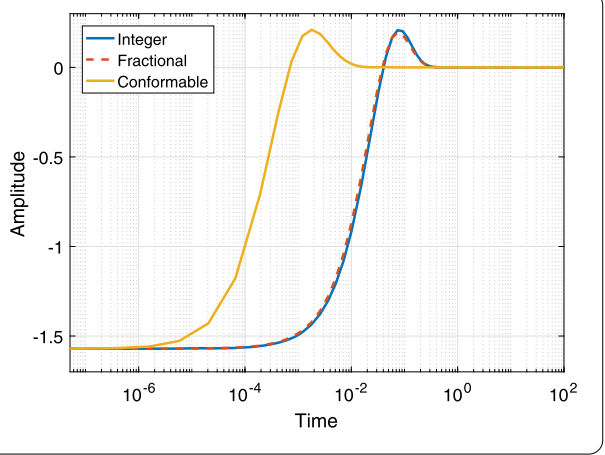

Figure 29 Comparison between $e_{2}$ obtained from the high-gain observer with integer, fractional, and GCD for the Van der Pol oscillator

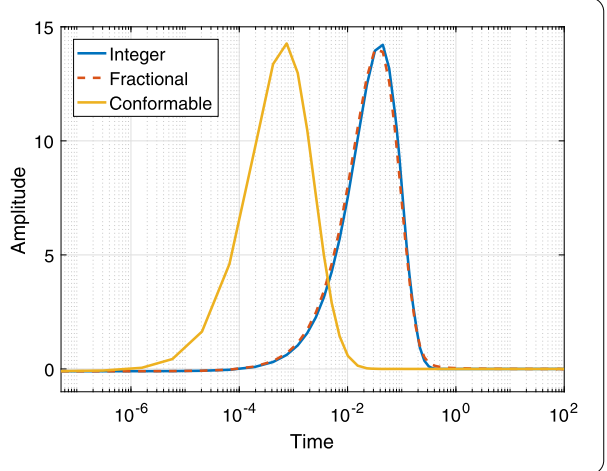

present finite-time stabilization, but with the GCD operator, the signals converge faster and smoother. Furthermore, Fig. 34 shows the phase portrait obtained with $\hat{x}_{1}$ and $\hat{x}_{2}$.

\section{Concluding remarks}

In this paper, an LQR-based estimator and a high-gain observer were proposed for a class of nonlinear systems with the general conformable derivative on their dynamics. By defining a general conformable exponential function, the estimators were proven to be finitetime stable in the sense of the derivative used by means of Lyapunov-like theorems. Then, to validate the proposed estimation scheme, simulations were performed on the general conformable models of the simple pendulum and the Van der Pol oscillator. Later, the results obtained with the conformable operator were compared with those obtained with the integer and fractional versions of the systems. 
Figure 30 Details of comparison between $e_{1}$ obtained from the high-gain observer with integer, fractional, and GCD for the Van der Pol oscillator

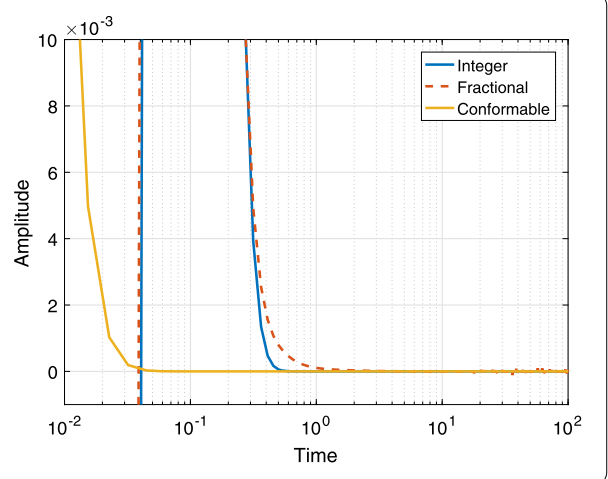

Figure 31 Details of comparison between $e_{2}$ obtained from the high-gain observer with integer, fractional, and GCD for the Van der Pol oscillator

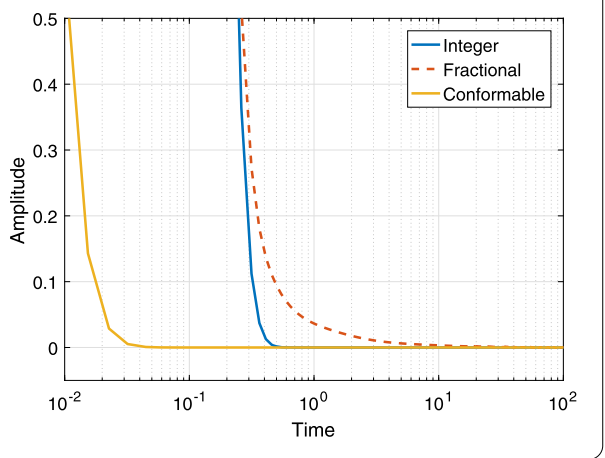

Figure 32 Comparison between the ISE for $e_{1}$ obtained from the high-gain observer with integer, fractional, and GCD for the Van der Pol oscillator

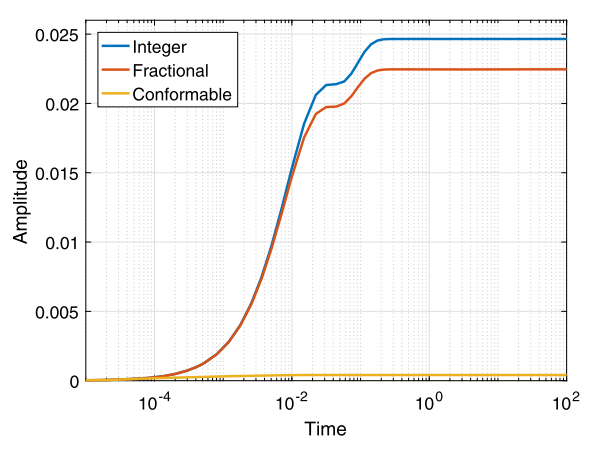

Regarding the results, the fractional case generally had a smaller overshoot than the integer and conformable cases; however, for the time scales shown, the signals in the fractional case did not reach the equilibrium. Furthermore, the integer and conformable cases presented finite-time stability, but the conformable case had a better performance than the integer one, showing a faster convergence though with a similar overshoot. Hence, as it was stated formerly, the fractional case did not present finite-time stability; the integer and conformable cases did, but the latter outperformed the former in the simulation results. In the graphs shown, it may be appreciated that, with the general conformable operator, the estimation error converges faster and the ISE is much smaller than with the other derivatives.

Comparing the estimators, both presented an acceptable performance with both systems, and their different outcomes depended on the choice of their parameters. 
Figure 33 Comparison between the ISE for $e_{2}$ obtained from the high-gain observer with integer, fractional, and GCD for the Van der Pol oscillator
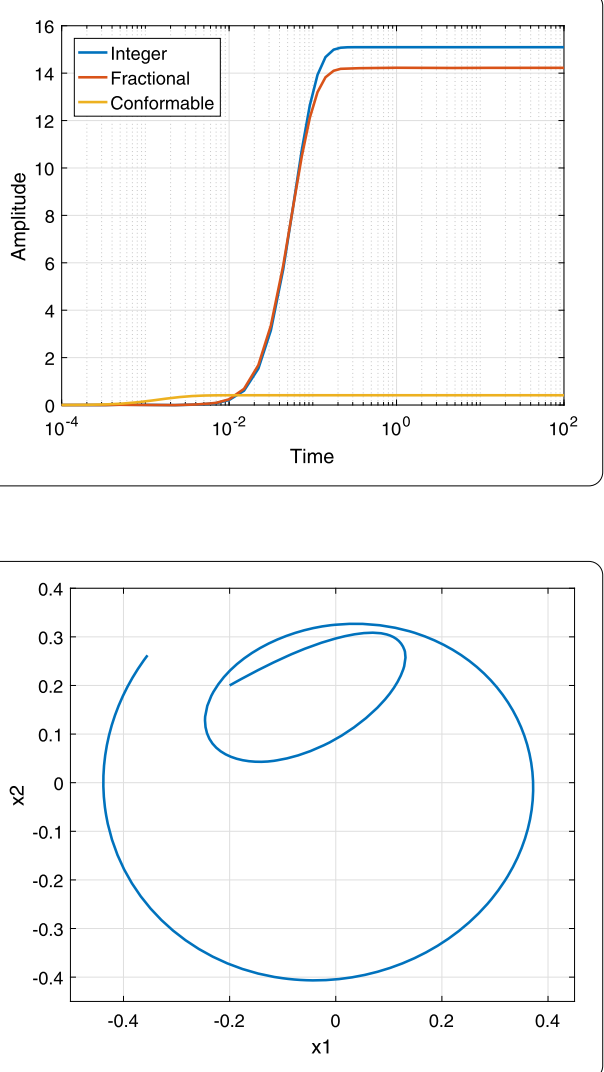

Figure 34 Phase portrait of the Van der Pol oscillator with the estimated states obtained from the high-gain observer

Finally, it is worth to note that these results may be improved through an adequate selection of the estimator gains and the conformable function $\psi(t, \alpha)$, which also depends on the system to which this estimation scheme is applied.

\section{Acknowledgements}

This work was supported by the National Council of Science and Technology (CONACYT) México as part of the Postdoctoral Project "Development of noninteger order derivatives and applications in nonlinear control systems" performed in the Universidad Iberoamericana at Mexico City.

\section{Funding}

Not applicable.

Availability of data and materials

Not applicable.

\section{Competing interests}

The authors declare that they have no competing interests.

\section{Authors' contributions}

All authors read and approved the final manuscript.

\section{Author details}

'Physics and Mathematics Department, Universidad Iberoamericana, Prol. Paseo de la Reforma 880, Lomas de Santa Fe, Álvaro Obregón, 01219 México City, México. ${ }^{2}$ Institute of Applied Research and Technology, Universidad Iberoamericana, Prol. Paseo de la Reforma 880, Lomas de Santa Fe, Álvaro Obregón, 01219 México City, México.

\section{Publisher's Note}

Springer Nature remains neutral with regard to jurisdictional claims in published maps and institutional affiliations. 


\section{References}

1. Tenreiro Machado, J.A., Silva, M.F., Barbosa, R.S., et al.: Some applications of fractional calculus in engineering. Math. Probl. Eng. 2010, 639801 (2010). https://doi.org/10.1155/2010/639801

2. Baleanu, D., Agarwal, R.P., Mohammadi, H., et al.: Some existence results for a nonlinear fractional differential equation on partially ordered Banach spaces. Bound. Value Probl. 2013, 112 (2013). https://doi.org/10.1186/1687-2770-2013-112

3. Baleanu, D., Rezapour, S., Mohammadi, H.: Some existence results on nonlinear fractional differential equations. Philos. Trans. R. Soc. Lond. A 371, 20120144 (2013). https://doi.org/10.1098/rsta.2012.0144

4. Sun, H., Zhang, Y., Baleanu, D., et al.: A new collection of real world applications of fractional calculus in science and engineering. Commun. Nonlinear Sci. Numer. Simul. 64, 213-231 (2018)

5. Goswami, A., Singh, J., Kumar, D., Sushila: An efficient analytical approach for fractional equal width equations describing hydro-magnetic waves in cold plasma. Physica A 524, 563-575 (2019)

6. Baleanu, D., Etemad, S., Rezapour, S.: A hybrid Caputo fractional modeling for thermostat with hybrid boundary value conditions. Bound. Value Probl. 2020, 64 (2020). https://doi.org/10.1186/s13661-020-01361-0

7. Srivastava, H.M., Dubey, V.P., Kumar, R., et al.: An efficient computational approach for a fractional-order biological population model with carrying capacity. Chaos Solitons Fractals 138, 109880 (2020). https://doi.org/10.1016/j.chaos.2020.109880

8. Veeresha, P., Prakasha, D.G., Kumar, D., et al.: An efficient computational technique for fractional model of generalized Hirota-Satsuma-coupled Korteweg-de Vries and coupled modified Korteweg-de Vries equations. J. Comput. Nonlinear Dyn. 15(7), 071003 (2020). https://doi.org/10.1115/1.4046898

9. Sales Teodoro, G., Tenreiro Machado, J.A., Capelas de Oliveira, E.: A review of definitions of fractional derivatives and other operators. J. Comput. Phys. 388, 195-208 (2019)

10. Baleanu, D., Fernández, A.: On fractional operators and their classifications. Mathematics 7(9), 830 (2019). https://doi.org/10.3390/math7090830

11. Caputo, M., Fabrizio, M.: A new definition of fractional derivative without singular kernel. Prog. Fract. Differ. Appl. 1, 73-85 (2015)

12. Atangana, A., Baleanu, D.: New fractional derivative without nonlocal and nonsingular kernel: theory and application to heat transfer model. Therm. Sci. 20, 763-769 (2016)

13. Baleanu, D., Mousalou, A., Rezapour, S.: On the existence of solutions for some infinite coefficient-symmetric Caputo-Fabrizio fractional integro-differential equations. Bound. Value Probl. 2017, 145 (2017) https://doi.org/10.1186/s13661-017-0867-9

14. Aydogan, M.S., Baleanu, D., Mousalou, A., et al.: On high order fractional integro-differential equations including the Caputo-Fabrizio derivative. Bound. Value Probl. 2018, 90 (2018). https://doi.org/10.1186/s13661-018-1008-9

15. Sheikh, N.A., Ali, F., Saqib, M., et al.: Comparison and analysis of the Atangana-Baleanu and Caputo-Fabrizio fractional derivatives for generalized Casson fluid model with heat generation and chemical reaction. Results Phys. 7, 789-800 (2017)

16. Syam, M.I., Al-Refai, M.: Fractional differential equations with Atangana-Baleanu fractional derivative: analysis and applications. Chaos Solitons Fractals 2, 100013 (2019). https://doi.org/10.1016/j.csfx.2019.100013

17. Kumar, D., Singh, J., Kumar, D., Baleanu, D.: On the analysis of vibration equation involving a fractional derivative with Mittag-Leffler law. Math. Methods Appl. Sci. 43(1), 443-457 (2020)

18. Khalil, R., Al Horani, M., Yousef, A., Sababheh, M.: A new definition of fractional derivative. J. Comput. Appl. Math. 264, 65-70 (2014)

19. Abdeljawad, T:: On conformable fractional calculus. J. Comput. Appl. Math. 279, 57-66 (2015)

20. Chung, W.S.: Fractional Newton mechanics with conformable fractional derivative. J. Comput. Appl. Math. 290 150-158 (2015)

21. Abdeljawad, T., Alzabut, J., Jarad, F.: A generalized Lyapunov-type inequality in the frame of conformable derivatives Adv. Differ. Equ. 2017, 321 (2017). https://doi.org/10.1186/s13662-017-1383-z

22. Zhou, H.W., Yang, S., Zhang, S.Q.: Conformable derivative approach to anomalous diffusion. Physica A 491, 1001-1013 (2018)

23. Bouaouid, M., Hilal, K., Melliani, S.: Nonlocal telegraph equation in frame of the conformable time-fractional derivative. Adv. Math. Phys. 2019, 7528937 (2019). https://doi.org/10.1155/2019/7528937

24. Khan, M.A., Gómez-Aguilar, J.F.: Tuberculosis model with relapse via fractional conformable derivative with power law. Math. Methods Appl. Sci. 42(18), 7113-7125 (2019)

25. Khan, T.U., Khan, M.A.: Generalized conformable fractional operators. J. Comput. Appl. Math. 346, 378-389 (2019)

26. Katugampola, U.N.: A new fractional derivative with classical properties. arXiv:1410.6535. arXiv preprint

27. Janaki, M., Elsayed, E.M., Kanagarajan, K.: Katugampola-type fractional differential equations with delay and impulses. Open Acc. J. Math. Theor. Phy. 1(3), 73-77 (2018)

28. Mahmudov, N.I., Emin, S.: Fractional-order boundary value problems with Katugampola fractional integral conditions. Adv. Differ. Equ. 2018, 81 (2018). https://doi.org/10.1186/s13662-018-1538-6

29. Salim, T.O., Abu Hany, A.A.K., El-Khatib, M.S.: On Katugampola Fourier transform. J. Math. 2019, 5942139 (2019). https://doi.org/10.1155/2019/5942139

30. Boucenna, D., Makhlouf, A.B., Hammami, M.A.: On Katugampola fractional order derivatives and Darboux problem for differential equations. CUBO 22(1), 125-136 (2020)

31. Akkurt, A., Yildirim, M.E., Yildirim, H.: A new generalized fractional derivative and integral. Konuralp J. Math. 5(2), 248-259 (2017)

32. Zhao, D., Luo, M.: General conformable fractional derivative and its physical interpretation. Calcolo 54, 903-917 (2017)

33. Bhat, S.P., Bernstein, D.S.: Finite-time stability of continuous autonomous systems. SIAM J. Control Optim. 38(3), $751-766$ (2000)

34. Moulay, E., Perruquetti, W.: Finite time stability and stabilization of a class of continuous systems. J. Math. Anal. Appl. 323, 1430-1443 (2006)

35. Moulay, E., Perruquetti, W.: Finite-time stability and stabilization: state of the art. In: Edwards, C., Fossas Colet, E., Fridman, L. (eds.) Advances in Variable Structure and Sliding Mode Control, pp. 23-41. Springer, Berlin (2006) 
36. Moulay, E., Perruquetti, W.: Finite time stability conditions for non autonomous continuous systems. Int. J. Control 81(5), 797-803 (2008)

37. Haimo, V.T.: Finite time controllers. SIAM J. Control Optim. 24(4), 760-770 (1986)

38. Amato, F., Ariola, M., Dorato, P.: Finite-time control of linear systems subject to parametric uncertainties and disturbances. Automatica 37(9), 1459-1463 (2001)

39. Yu, X., Yin, J., Khoo, S.: New Lyapunov conditions of stochastic finite-time stability and instability of nonlinear time-varying SDEs. Int. J. Control 2019 (2019). https://doi.org/10.1080/00207179.2019.1662948

40. Lazarević, M.P., Spasić, A.: Finite-time stability analysis of fractional order time-delay systems: Gronwall's approach. Math. Comput. Model. 49(3-4), 475-481 (2009)

41. Lu, Q., Zhu, Y.: Finite-time stability of uncertain fractional difference equations. Fuzzy Optim. Decis. Mak. 19, 239-249 (2020)

42. Thanh, N.T., Phat, V.N., Niamsup, P.: New finite-time stability analysis of singular fractional differential equations with time-varying delay. Fract. Calc. Appl. Anal. 23(2), 504-519 (2020)

43. Makhlouf, A.B., Naifar, O., Hammami, M.A., Wu, B.F.T.: FTB of conformable fractional order linear systems. Math. Probl. Eng. 2018, 2572986 (2018). https://doi.org/10.1155/2018/2572986

44. Jmal, A., Makhlouf, A.B., Nagy, A.M., Naifar, O.: Finite-time stability for Caputo-Katugampola fractional-order time-delayed neural networks. Neural Process. Lett. 50, 607-621 (2019)

45. Makhlouf, A.B., Nagy, A.M.: Finite-time stability of linear Caputo-Katugampola fractional-order time delay systems. Asian J. Control 21(6), 1-10 (2019)

46. Shen, J., Lam, J.: Non-existence of finite-time stable equilibria in fractional-order nonlinear systems. Automatica 50, 547-551 (2014)

47. Muñóz-Vázquez, A.J., Sánchez-Orta, A., Parra-Vega, V.: A general result on non-existence of finite-time stable equilibria in fractional-order systems. J. Franklin Inst. 356(1), 268-275 (2019)

48. Botelho, F.: Functional Analysis and Applied Optimization in Banach Spaces: Applications to Non-convex Variational Models. Springer, Switzerland (2014)

49. Troutman, J.L.: Variational Calculus and Optimal Control: Optimization with Elementary Convexity. Springer, New York (1996)

50. Martínez Fuentes, O., Martínez Guerra, R.: A novel Mittag-Leffler stable estimator for nonlinear fractional-order systems: a linear quadratic regulator approach. Nonlinear Dyn. 94(3), 1973-1986 (2018)

51. Martínez Fuentes, O., Martínez Guerra, R.: A high-gain observer with Mittag-Leffler rate of convergence for a class of nonlinear fractional-order systems. Commun. Nonlinear Sci. Numer. Simul. 79, 104909 (2019). https://doi.org/10.1016/j.cnsns.2019.104909

52. Valério, D.: MATLAB central file exchange. Retrieved March 26, 2020. https://www.mathworks.com/matlabcentral/fileexchange/8312-ninteger

53. Anli, E., Ozkol, l.: Classical and fractional-order analysis of the free and forced double pendulum. Engineering 2 , 935-949 (2010)

54. David, S.A., Valentim, C.A. Jr.: Fractional Euler-Lagrange equations applied to oscillatory systems. Mathematics 3 258-272 (2015)

55. N'Doye, I., Laleg Kirati, T.: Stability and trajectories analysis of a fractional generalization of simple pendulum dynamic equation. In: The 2019 18th European Control Conference (ECC), Napoli, Italy, June 2019, pp. 25-28 (2019)

56. Van der Pol, B.: A theory of the amplitude of free and forced triode vibrations. Radiol. Rev. 1, $701-710$ (1920)

57. Barbosa, R.S., Tenreiro Machado, J.A., Ferreira, I.M., Tar, J.K.: Dynamics of the fractional-order Van der Pol oscillator. In: Second IEEE International Conference on Computational Cybernetics, ICCC 2004, Vienna, pp. 373-378 (2004)

58. Barbosa, R.S., Tenreiro Machado, J.A., Vinagre, B.M., Calderón, A.J.: Analysis of the Van der Pol oscillator containing derivatives of fractional order. J. Vib. Control 13(9-10), 1291-1301 (2007)

59. Petráš, I.: Fractional-Order Nonlinear Systems: Modeling, Analysis and Simulation. Springer, Beijing (2011)

\section{Submit your manuscript to a SpringerOpen ${ }^{\circ}$ journal and benefit from:}

- Convenient online submission

- Rigorous peer review

- Open access: articles freely available online

- High visibility within the field

- Retaining the copyright to your article

Submit your next manuscript at $\boldsymbol{s p r i n g e r o p e n . c o m ~}$ 